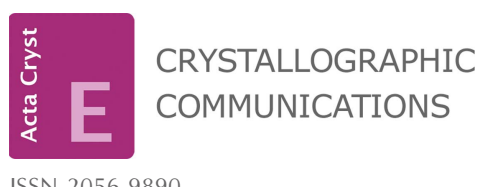

ISSN 2056-9890

Received 6 April 2020

Accepted 29 April 2020

Edited by A. J. Lough, University of Toronto, Canada

Keywords: crystal structure; perimidine; phenol; Hirshfeld surface.

CCDC reference: 1976884

Supporting information: this article has supporting information at journals.iucr.org/e

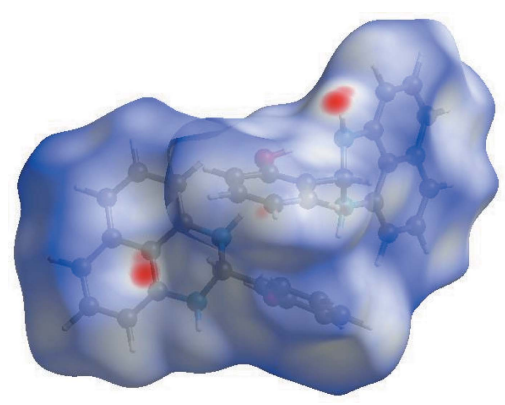

\section{Crystal structure, Hirshfeld surface analysis and DFT studies of 2-(2,3-dihydro-1 $\mathrm{H}$-perimidin-2- yl)phenol}

\author{
Ballo Daouda, ${ }^{\mathrm{a}, \mathrm{b} *}$ Nanou Tiéba Tuo, ${ }^{\mathrm{c}}$ Niameke Jean-Baptiste Kangah, ${ }^{\mathrm{c}}$ Tuncer \\ Hökelek, ${ }^{\mathrm{d}}$ Charles Guillaume Kodjo, ${ }^{\mathrm{c}}$ Pascal Retailleau ${ }^{\mathrm{e}}$ and El Mokhtar Essassi ${ }^{\mathrm{a}}$
}

\author{
aLaboratoire de Chimie Organique Heterocyclique URAC 21, Pôle de Competence Pharmacochimie, Faculté des \\ Sciences, Université Mohammed V, Rabat, Morocco, 'baboratoire de Chimie Organique et de Substances Naturelles, \\ UFR Sciences des Structures de la Matière et Technologie, Université Félix Houphouët-Boigny, 22 BP 582 Abidjan, Côte \\ d'Ivoire, 'Laboratoire de Thermodynamique et Physicochimie du Milieu, Université Nangui, Abrogoua, UFR-SFA, 02 BP \\ 801 Abidjan 02, Côte d'Ivoire, 'd Department of Physics, Hacettepe University, 06800 Beytepe, Ankara, Turkey, and \\ e Institut de Chimie des Substances Naturelles, 1 av. de la Terrasse, 91198 Gif sur Yvette, France. *Correspondence e-mail: \\ daoudaballo526@gmail.com
}

The asymmetric unit of the title compound, $\mathrm{C}_{17} \mathrm{H}_{14} \mathrm{~N}_{2} \mathrm{O}$, contains two independent molecules each consisting of perimidine and phenol units. The tricyclic perimidine units contain naphthalene ring systems and non-planar $\mathrm{C}_{4} \mathrm{~N}_{2}$ rings adopting envelope conformations with the $\mathrm{C}$ atoms of the NCN groups hinged by 44.11 (7) and $48.50(6)^{\circ}$ with respect to the best planes of the other five atoms. Intramolecular $\mathrm{O}-\mathrm{H} \cdots \mathrm{N}$ hydrogen bonds may help to consolidate the molecular conformations. The two independent molecules are linked through an $\mathrm{N}-\mathrm{H} \cdots \mathrm{O}$ hydrogen bond. The Hirshfeld surface analysis of the crystal structure indicates that the most important contributions for the crystal packing are from $\mathrm{H} \cdots \mathrm{H}(52.9 \%)$ and $\mathrm{H} \cdots \mathrm{C} / \mathrm{C} \cdots \mathrm{H}(39.5 \%)$ interactions. Hydrogen bonding and van der Waals interactions are the dominant interactions in the crystal packing. Density functional theory (DFT) optimized structures at the B3LYP/ 6-311 G(d,p) level are compared with the experimentally determined molecular structure in the solid state. The HOMO-LUMO behaviour was elucidated to determine the energy gap.

\section{Chemical context}

1- $H$ Perimidines are defined as peri-naphtho-fused pyrimidines (Varsha et al., 2010). They were first discovered in 1874 (De Aguiar, 1874) and are characterized either by a binding deficit or an excess of $\pi$ binding (Woodgate et al., 1987). They are used as intermediates in dyes, dyeing and polymerization systems (Watanab et al., 1977) and have been recognized as new carbene ligands (Bazinet et al., 2003), attracting great interest (Bu et al., 2001; Starshikoy et al., 1973). 1- $H$ Perimidines also exhibit important biological activities (Zhou et al., 2019), having the potential to act as anti-inflammatory agents (Zhang et al., 2017) and inhibitors of enzymes (Alam et al., 2016) and to have applications in fluorescence (Giani et al., 2016), catalysis (Behbahani et al., 2017), corrosion inhibition (He et al., 2018) and in coordination chemistry (Booysen et al., 2016; Mahapatra et al., 2015).

Perimidines are obtained by the condensation of 1,8-diaminonaphthalene with various carbonyl groups. As a continuation of our research into the development of new perimidine derivatives with potential pharmacological appli- 
cations, we have studied the reaction of the condensation of salicylaldehyde and 1,8- diaminonaphthalene in ether under agitation at room temperature to give the title compound in good yield. The title compound was obtained for the first time and characterized by single-crystal X-ray diffraction techniques as well as by Hirshfeld surface analysis. The results of the calculations by density functional theory (DFT), carried out at the B3LYP/6-311G (d,p) level, are compared with the experimentally determined molecular structure in the solid state.

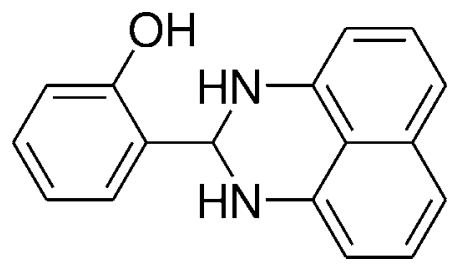

\section{Structural commentary}

The asymmetric unit of the title compound, I, contains two crystallographically independent molecules each consisting of perimidine and phenol units, where the tricyclic perimidine units contain naphthalene ring systems and non-planar $\mathrm{C}_{4} \mathrm{~N}_{2}$ rings (Fig. 1). A puckering analysis of the non-planar sixmembered $\mathrm{C}_{4} \mathrm{~N}_{2}, B(\mathrm{~N} 1 A / \mathrm{N} 2 A / \mathrm{C} 1 A / \mathrm{C} 9 A-\mathrm{C} 11 A)$ and $B^{\prime}$ $(\mathrm{N} 1 A / \mathrm{N} 2 A / \mathrm{C} 1 A / \mathrm{C} 9 B-\mathrm{C} 11 B)$ rings gave the parameters $q_{2}=$

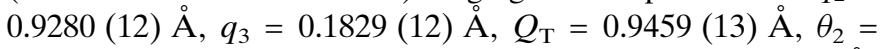
$75.85(15)^{\circ}$ and $\varphi_{2}=134.47(18)^{\circ}$ for $B$ and $q_{2}=0.5320$ (11) $\AA$, $q_{3}=0.3791(11) \AA, Q_{\mathrm{T}}=0.6533(14) \AA, \theta_{2}=54.33(12)^{\circ}$ and $\varphi$ ${ }_{2}=-5.47(13)^{\circ}$ for $B^{\prime}$; both rings adopt envelope conformations, where atoms $\mathrm{C} 1 A$ and $\mathrm{C} 1 B$ are at the flap positions and at distances of 0.6044 (12) and -0.6590 (13) A, respectively, from the best planes through the other five atoms. The $\mathrm{C}_{4} \mathrm{~N}_{2}$ rings may alternatively be described as being hinged about the $\mathrm{N} \cdots \mathrm{N}$ vectors with the $\mathrm{N} 1 A / \mathrm{C} 1 A / \mathrm{N} 2 A$ and $\mathrm{N} 1 B / \mathrm{C} 1 B / \mathrm{N} 2 B$ planes being inclined by 44.11 (7) and $48.50(6)^{\circ}$, respectively,

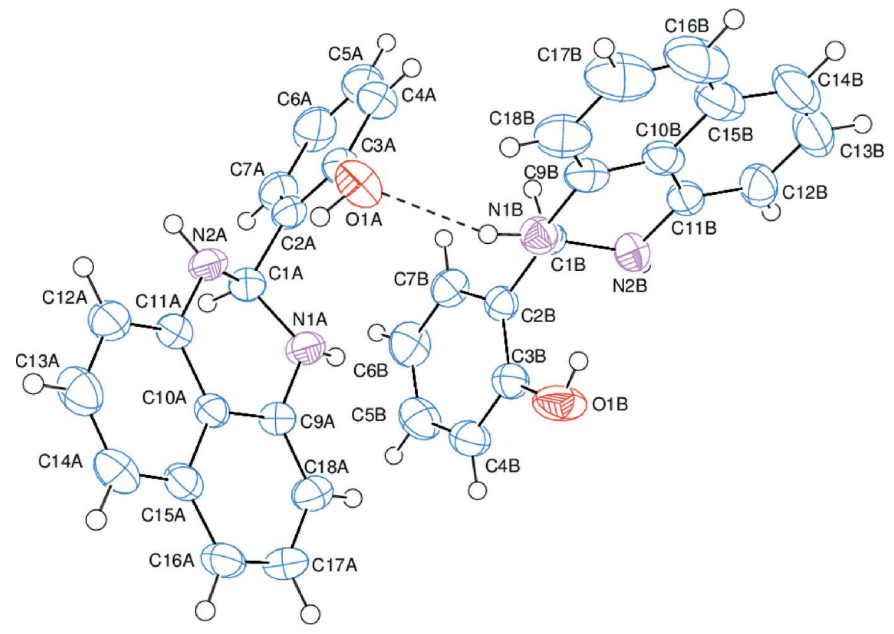

Figure 1

The asymmetric unit of the title compound with the atom-numbering scheme. Displacement ellipsoids are drawn at the $50 \%$ probability level.
Table 1

Hydrogen-bond geometry $\left(\AA{ }^{\circ}\right)$.

\begin{tabular}{lllll}
\hline$D-\mathrm{H} \cdots A$ & $D-\mathrm{H}$ & $\mathrm{H} \cdots A$ & $D \cdots A$ & $D-\mathrm{H} \cdots A$ \\
\hline $\mathrm{O} 1 A-\mathrm{H} 1 O A \cdots \mathrm{N} 1 A$ & $0.86(2)$ & $2.66(2)$ & $3.1072(16)$ & $113.8(17)$ \\
$\mathrm{O} 1 A-\mathrm{H} 1 O A \cdots \mathrm{N} 2 A$ & $0.86(2)$ & $2.03(2)$ & $2.7763(16)$ & $144.6(19)$ \\
$\mathrm{O} 1 B-\mathrm{H} 1 O B \cdots \mathrm{N} 1 B$ & $0.84(2)$ & $2.20(3)$ & $2.8835(16)$ & $138(2)$ \\
$\mathrm{O} 1 B-\mathrm{H} 1 O B \cdots \mathrm{N} 2 B$ & $0.84(2)$ & $2.47(2)$ & $3.0196(16)$ & $123(2)$ \\
$\mathrm{N} 1 B-\mathrm{H} 1 N B \cdots \mathrm{O} 1 A$ & $0.865(17)$ & $2.331(17)$ & $3.1608(18)$ & $160.8(14)$ \\
\hline
\end{tabular}

to the best planes through the other five atoms $(\mathrm{N} 1 A / \mathrm{N} 2 A$ / $\mathrm{C} 9 A-\mathrm{C} 11 A)$ and $(\mathrm{N} 1 B / \mathrm{N} 2 B / \mathrm{C} 9 B-\mathrm{C} 11 B)$. Rings $A(\mathrm{C} 2 A-$ $\mathrm{C} 7 A), C(\mathrm{C} 10 A-\mathrm{C} 15 A), D(\mathrm{C} 9 A / \mathrm{C} 10 A / \mathrm{C} 15 A-\mathrm{C} 18 A)$ and $A^{\prime}$ $(\mathrm{C} 2 B-\mathrm{C} 7 B), C^{\prime}(\mathrm{C} 10 B-\mathrm{C} 15 B), D^{\prime}(\mathrm{C} 9 B / \mathrm{C} 10 B / \mathrm{C} 15 B-\mathrm{C} 18 B)$ are oriented at dihedral angles of $A / C=76.78(4), A / D=$ $78.49(4), C / D=2.09(3)^{\circ}$ and $A^{\prime} / C^{\prime}=88.43(3), A^{\prime} / D^{\prime}=$ $88.31(3), \quad C^{\prime} / D^{\prime}=3.26(4)^{\circ}$. Intramolecular $\mathrm{O}-\mathrm{H} \cdots \mathrm{N}$ hydrogen bonds (Table 1) may be effective in consolidating the conformations of the two independent molecules.

\section{Supramolecular features}

In the crystal, the two molecules in the asymmetric unit are linked through an $\mathrm{N}-\mathrm{H} \cdots \mathrm{O}$ hydrogen bond (Table 1, Fig. 1).

\section{Hirshfeld surface analysis}

In order to visualize the intermolecular interactions in the crystal of the title compound, a Hirshfeld surface (HS) analysis (Hirshfeld, 1977; Spackman \& Jayatilaka, 2009) was carried out by using Crystal Explorer 17.5 (Turner et al., 2017). In the HS plotted over $d_{\text {norm }}$ (Fig. 2), the white surface indicates contacts with distances equal to the sum of van der Waals radii, and the red and blue colours indicate distances shorter (in close contact) or longer (distinct contact) than the van der Waals radii, respectively (Venkatesan et al., 2016). The brightred spots indicate their roles as the respective donors and/or acceptors.

The shape-index of the HS is a tool to visualize the $\pi-\pi$ stacking by the presence of adjacent red and blue triangles; if

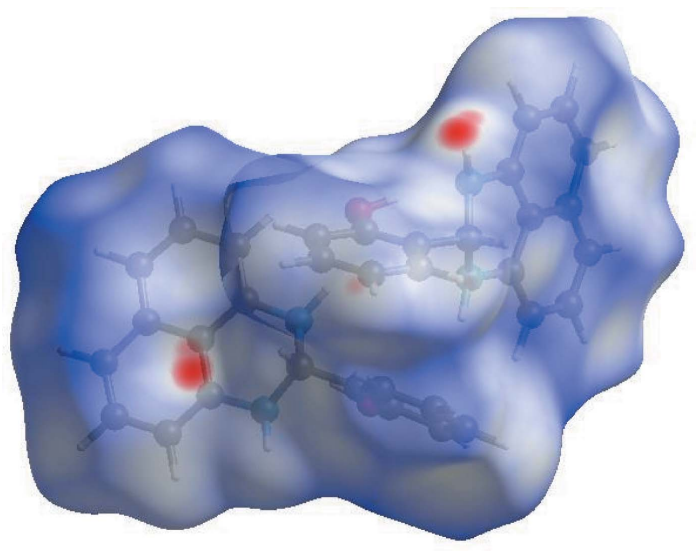

Figure 2

View of the three-dimensional Hirshfeld surface of the title compound plotted over $d_{\text {norm }}$ in the range -0.1813 to 1.6330 a.u. 


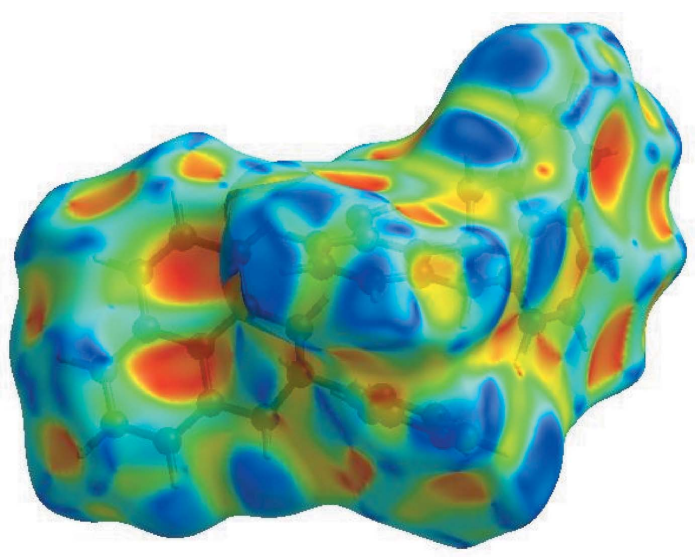

Figure 3

Hirshfeld surface of the title compound plotted over shape-index.

there are no adjacent red and/or blue triangles, then there are no $\pi-\pi$ interactions. Fig. 3 clearly suggests that there are no $\pi-$ $\pi$ interactions in $\mathbf{I}$. The overall two-dimensional fingerprint plot (McKinnon et al., 2007) is shown in Fig. 4a, and those delineated into $\mathrm{H} \cdots \mathrm{H}, \mathrm{H} \cdots \mathrm{C} / \mathrm{C} \cdots \mathrm{H}, \mathrm{H} \cdots \mathrm{O} / \mathrm{O} \cdots \mathrm{H}, \mathrm{H} \cdots \mathrm{N} /$ $\mathrm{N} \cdots \mathrm{H}$ and $\mathrm{C} \cdots \mathrm{C}$ contacts are illustrated in Fig. $4 b-f$, respectively, together with their relative contributions to the Hirshfeld surface. The most important interaction is $\mathrm{H} \cdots \mathrm{H}$, contributing $52.9 \%$ to the overall crystal packing, which is reflected in Fig. $4 b$ as widely scattered points of high density due to the large hydrogen content of the molecule, with the tip at $d_{\mathrm{e}}=d_{\mathrm{i}}=1.10 \AA$. The pair of characteristic wings in the fingerprint plot delineated into $\mathrm{H} \cdots \mathrm{C} / \mathrm{C} \cdots \mathrm{H}$ contacts, Fig. $4 c$, ( $39.5 \%$ contribution to the HS) have the tips at $d_{\mathrm{e}}+d_{\mathrm{i}}=$ $2.50 \AA$. The scattered points in the pair of spikes in the fingerprint plot delineated into $\mathrm{H} \cdots \mathrm{O} / \mathrm{O} \cdots \mathrm{H}$ (Fig. $4 d, 5.7 \%$ contribution) have a symmetrical distribution with the tips at $d_{\mathrm{e}}+d_{\mathrm{i}}=2.49 \AA$. The $\mathrm{H} \cdots \mathrm{N} / \mathrm{N} \cdots \mathrm{H}$ contacts (Fig. $4 e, 1.3 \%$ contribution) have a distribution of points with the tips at
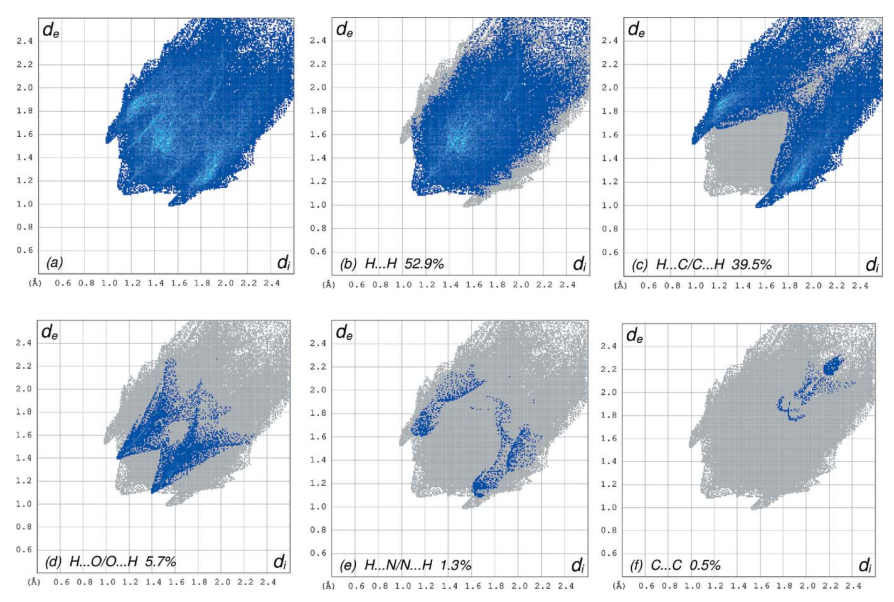

Figure 4

The full two-dimensional fingerprint plots for the title compound, showing $(a)$ all interactions, and delineated into $(b) \mathrm{H} \cdots \mathrm{H},(c) \mathrm{H} \cdots \mathrm{C} /$ $\mathrm{C} \cdots \mathrm{H}, \quad(d) \quad \mathrm{H} \cdots \mathrm{O} / \mathrm{O} \cdots \mathrm{H}, \quad(e) \quad \mathrm{H} \cdots \mathrm{N} / \mathrm{N} \cdots \mathrm{H}$ and $(f) \quad \mathrm{O} \cdots \mathrm{C} / \mathrm{C} \cdots \mathrm{O}$ interactions. The $d_{\mathrm{i}}$ and $d_{\mathrm{e}}$ values are the closest internal and external distances (in $\AA$ ) from given points on the Hirshfeld surface contacts.

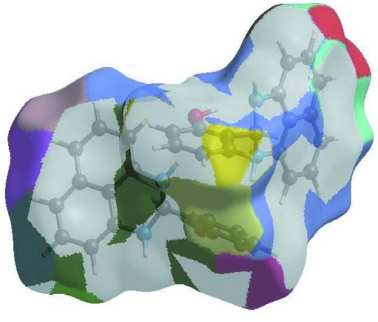

(a)

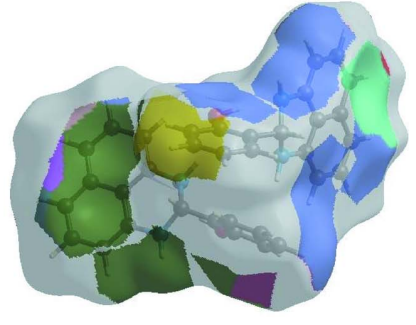

(b)

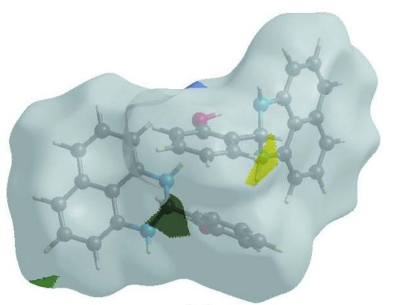

(c)

Figure 5

The Hirshfeld surface representations with the function $d_{\text {norm }}$ plotted onto the surface for $(a) \mathrm{H} \cdots \mathrm{H},(b) \mathrm{H} \cdots \mathrm{C} / \mathrm{C} \cdots \mathrm{H}$ and $(c) \mathrm{H} \cdots \mathrm{O} / \mathrm{O} \cdots \mathrm{H}$ interactions.

$d_{\mathrm{e}}+d_{\mathrm{i}}=2.72 \AA$. Finally, the C...C interactions $(0.5 \%$ contribution to the overall crystal packing) are reflected in Fig. $4 f$ as low density wings with the tips at $d_{\mathrm{e}}+d_{\mathrm{i}}=3.60 \AA$.

The Hirshfeld surface representations with the function $d_{\text {norm }}$ plotted onto the surface are shown for the $\mathrm{H} \cdots \mathrm{H}$, $\mathrm{H} \cdots \mathrm{C} / \mathrm{C} \cdots \mathrm{H}$ and $\mathrm{H} \cdots \mathrm{O} / \mathrm{O} \cdots \mathrm{H}$ interactions in Fig. $5 a-c$, respectively.

The Hirshfeld surface analysis confirms the importance of $\mathrm{H}$-atom contacts in establishing the packing. The large number of $\mathrm{H} \cdots \mathrm{H}$ and $\mathrm{H} \cdots \mathrm{C} / \mathrm{C} \cdots \mathrm{H}$ interactions suggest that van der Waals interactions and hydrogen bonding play the major roles in the crystal packing (Hathwar et al., 2015).

\section{DFT calculations}

The optimized structure of the title compound, $\mathbf{I}$, in the gas phase was generated theoretically via density functional theory (DFT) using standard B3LYP functional and 6-311 G(d,p) basis-set calculations (Becke, 1993) as implemented in GAUSSIAN 09 (Frisch et al., 2009). The theoretical and experimental results were in good agreement (Table 2). The highest-occupied molecular orbital (HOMO), acting as an electron donor, and the lowest-unoccupied molecular orbital (LUMO), acting as an electron acceptor, are very important parameters for quantum chemistry. When the energy gap is small, the molecule is highly polarizable and has high chemical reactivity. The DFT calculations provide some important information on the reactivity and site selectivity of the molecular framework. $E_{\text {Hомо }}$ and $E_{\mathrm{LUMO}}$, which clarify the inevitable charge-exchange collaboration inside the studied material, electronegativity $(\chi)$, hardness $(\eta)$, potential $(\mu)$, electrophilicity $(\omega)$ and softness $(\sigma)$ are recorded in Table 3. The significance of $\eta$ and $\sigma$ is for the evaluation of both the reactivity and stability. The electron transition from the 
Table 2

Comparison of selected X-ray and DFT geometrical parameters $\left(\AA{ }^{\circ}\right)$.

\begin{tabular}{lll}
\hline Bonds/angles & X-ray & B3LYP/6-311G(d,p) \\
\hline $\mathrm{C} 1 A-\mathrm{N} 1 A$ & $1.4597(17)$ & 1.40941 \\
$\mathrm{C} 1 A-\mathrm{N} 2 A$ & $1.4646(19)$ & 1.35557 \\
$\mathrm{C} 1 A-\mathrm{C} 2 A$ & $1.5079(17)$ & 1.43731 \\
$\mathrm{C} 1 A-\mathrm{H} 1 A$ & 0.9800 & 1.03211 \\
$\mathrm{~N} 1 A-\mathrm{C} 9 A$ & $1.3944(17)$ & 1.42420 \\
$\mathrm{~N} 1 A-\mathrm{H} 1 N 1$ & $0.873(19)$ & 1.00630 \\
$\mathrm{O} 1 A-\mathrm{C} 3 A$ & $1.3693(18)$ & 1.40953 \\
$\mathrm{O} 1 A-\mathrm{H} 1 \mathrm{O} A$ & $0.86(2)$ & 0.97032 \\
$\mathrm{C} 2 A-\mathrm{C} 7 A$ & $1.388(2)$ & 1.42763 \\
$\mathrm{C} 2 A-\mathrm{C} 3 A$ & $1.3923(19)$ & 1.42630 \\
$\mathrm{~N} 2 A-\mathrm{C} 11 A$ & $1.4081(17)$ & 1.36897 \\
$\mathrm{~N} 1 A-\mathrm{C} 1 A-\mathrm{N} 2 A$ & & \\
$\mathrm{~N} 1 A-\mathrm{C} 1 A-\mathrm{C} 2 A$ & $106.61(11)$ & 115.07 \\
$\mathrm{~N} 2 A-\mathrm{C} 1 A-\mathrm{C} 2 A$ & $110.09(11)$ & 125.03 \\
$\mathrm{~N} 1 A-\mathrm{C} 1 A-\mathrm{H} 1 A$ & $109.23(11)$ & 109.89 \\
$\mathrm{~N} 2 A-\mathrm{C} 1 A-\mathrm{H} 1 A$ & 110.3 & 110.17 \\
$\mathrm{C} 2 A-\mathrm{C} 1 A-\mathrm{H} 1 A$ & 110.3 & 110.03 \\
$\mathrm{C} 9 A-\mathrm{N} 1 A-\mathrm{C} 1 A$ & 110.3 & 110.08 \\
$\mathrm{C} 9 A-\mathrm{N} 1 A-\mathrm{H} 1 N 1$ & $117.08(11)$ & 117.82 \\
$\mathrm{C} 3 A-\mathrm{O} 1 A-\mathrm{H} 1 O A$ & $115.0(12)$ & 114.98 \\
& $106.1(14)$ & 107.84 \\
& &
\end{tabular}

HOMO to the LUMO energy level is shown in Fig. 6. The HOMO and LUMO are localized in the plane extending from the whole 2-(2,3-dihydro- $1 H$-perimidin-2-yl)phenol ring. The energy band gap [ $\left.\Delta E=E_{\mathrm{LUMO}}-E_{\mathrm{HOMO}}\right]$ of the molecule is $1.4933 \mathrm{eV}$, the frontier molecular orbital energies $E_{\text {HOMO }}$ and $E_{\mathrm{LUMO}}$ being -3.2606 and $-1.7673 \mathrm{eV}$, respectively.

\section{Database survey}

Similar perimidine derivatives have also been reported in which the groups at position 2 are almost coplanar with the perimidic nucleus. Examples related to the title compound, I, are II (Ghorbani, 2012), III (Fun et al., 2011), IV (Maloney et al., 2013), V (Cucciolito et al., 2013) and VI (Manimekalai et al., 2014), where III and V are most closely related while II, IV and $\mathbf{V I}$ are more distant relatives.

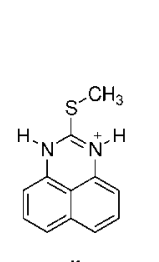

II

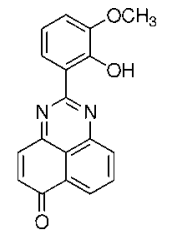

III

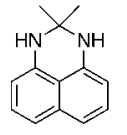

IV

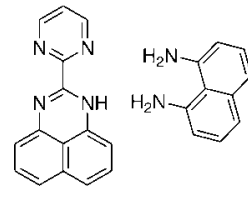

$\mathrm{v}$

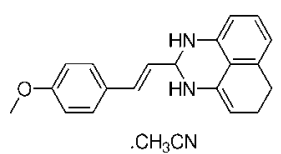

v

\section{Synthesis and crystallization}

$0.35 \mathrm{~mol}(1.48 \mathrm{~g})$ of 1,8 -diaminonaphthalene and $18.8 \mathrm{mmol}$ $(2 \mathrm{ml})$ of salicylaldehyde were introduced into a $250 \mathrm{ml}$ flask and $30 \mathrm{ml}$ of ether were added thereto. The mixture was
Table 3

Calculated energies.

\begin{tabular}{lr}
\hline Molecular Energy (a.u.) (eV) & Compound I \\
\hline Total Energy $T E(\mathrm{eV})$ & -22880.3725 \\
$E_{\mathrm{HOMO}}(\mathrm{eV})$ & -3.2606 \\
$E_{\mathrm{LUMO}}(\mathrm{eV})$ & -1.7673 \\
Gap, $\Delta E(\mathrm{eV})$ & 1.4933 \\
Dipole moment, $\mu$ (Debye) & 3.3491 \\
Ionization potential, $I(\mathrm{eV})$ & 3.2606 \\
Electron affinity, $A$ & 1.7673 \\
Electronegativity, $\chi$ & 2.5139 \\
Hardness, $\eta$ & 0.7466 \\
Electrophilicity index, $\omega$ & 4.2322 \\
Softness, $\sigma$ & 1.3393 \\
Fraction of electron transferred, $\Delta N$ & 3.0042 \\
\end{tabular}

stirred magnetically for 3 days. The grey precipitate that formed was recovered by filtration, washed with ether, rinsed with ethanol and dried under Büchner. The resulting brownish powder was recrystallized several times from ethanol to obtain colourless 2-(2,3-dihydro-1H-perimidin-2-yl)phenol product $\left(R_{\mathrm{f}}=0.70\right.$ in hexane/ethyl acetate (1:0.5), yield: $97 \% \mathrm{~A}$ significant quantity of the colourless monocrystalline product was obtained by the slow evaporation of the solvent after 15 days.

\section{Refinement}

Crystal data, data collection and structure refinement details are summarized in are summarized in Table 4. The $\mathrm{H}$ atoms of $\mathrm{OH}$ and $\mathrm{NH}$ groups were located in difference-Fourier maps and refined freely. The $\mathrm{C}$-bound $\mathrm{H}$ atoms were positioned geometrically, with $\mathrm{C}-\mathrm{H}=0.93 \AA$ (for aromatic $\mathrm{H}$ atoms) and $0.98 \AA$ (for methine $\mathrm{H}$ atom) and constrained to ride on their parent atoms, with $U_{\text {iso }}(\mathrm{H})=1.2 U_{\text {eq }}(\mathrm{C})$.

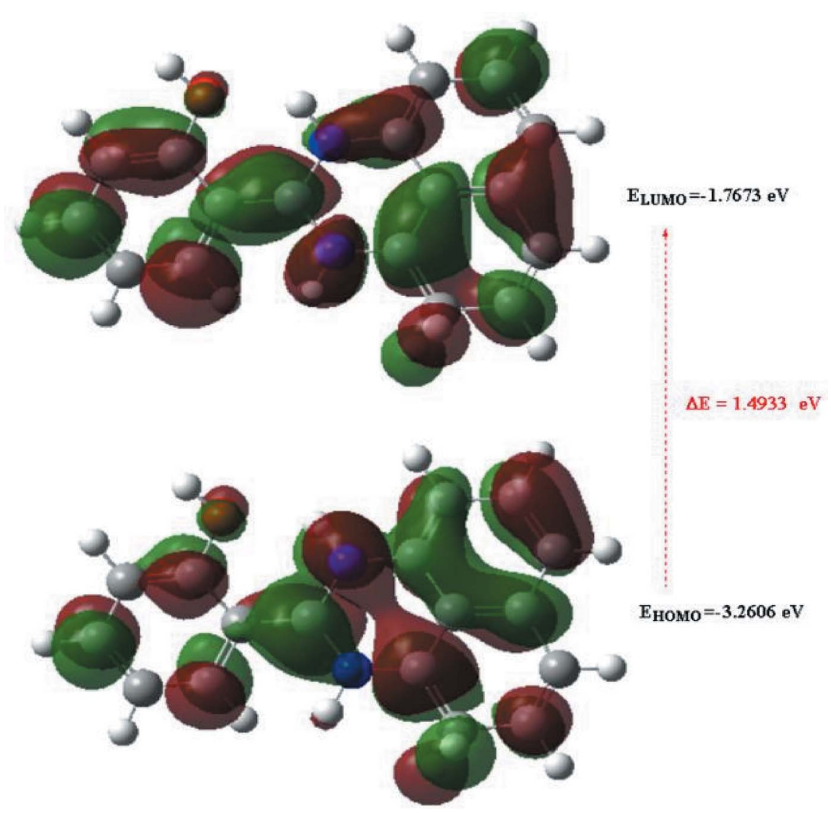

Figure 6

The energy band gap of the title compound. 
Table 4

Experimental details.

\begin{tabular}{|c|c|}
\hline \multicolumn{2}{|l|}{ Crystal data } \\
\hline Chemical formula & $\mathrm{C}_{17} \mathrm{H}_{14} \mathrm{~N}_{2} \mathrm{O}$ \\
\hline$M_{\mathrm{r}}$ & 262.30 \\
\hline Crystal system, space group & Monoclinic, $P 2_{1} / c$ \\
\hline Temperature $(\mathrm{K})$ & 293 \\
\hline$a, b, c(\AA)$ & $\begin{array}{l}9.0710(4), 12.0526(7) \\
24.6120(11)\end{array}$ \\
\hline$\beta\left({ }^{\circ}\right)$ & $95.999(4)$ \\
\hline$V\left(\AA^{6}\right)$ & $2676.1(2)$ \\
\hline$Z$ & 8 \\
\hline Radiation type & Мо $K \alpha$ \\
\hline$\mu\left(\mathrm{mm}^{-1}\right)$ & 0.08 \\
\hline Crystal size (mm) & $0.60 \times 0.35 \times 0.05$ \\
\hline \multicolumn{2}{|l|}{ Data collection } \\
\hline Diffractometer & Rigaku XtaLAB PRO \\
\hline Absorption correction & $\begin{array}{l}\text { Multi-scan (CrysAlis PRO; Rigaku } \\
\text { OD, 2018) }\end{array}$ \\
\hline$T_{\min }, T_{\max }$ & $0.212,1.000$ \\
\hline $\begin{array}{l}\text { No. of measured, independent and } \\
\text { observed }[I>2 \sigma(I)] \text { reflections }\end{array}$ & $29344,6395,4554$ \\
\hline$R_{\mathrm{int}}$ & 0.042 \\
\hline$(\sin \theta / \lambda)_{\max }\left(\AA^{-1}\right)$ & 0.690 \\
\hline \multicolumn{2}{|l|}{ Refinement } \\
\hline$R\left[F^{2}>2 \sigma\left(F^{2}\right)\right], w R\left(F^{2}\right), S$ & $0.046,0.120,1.03$ \\
\hline No. of reflections & 6395 \\
\hline No. of parameters & 379 \\
\hline $\mathrm{H}$-atom treatment & $\begin{array}{l}\mathrm{H} \text { atoms treated by a mixture of } \\
\text { independent and constrained } \\
\text { refinement }\end{array}$ \\
\hline$\Delta \rho_{\max }, \Delta \rho_{\min }\left(\mathrm{e} \AA^{-3}\right)$ & $0.19,-0.21$ \\
\hline
\end{tabular}

Computer programs: CrysAlis PRO (Rigaku OD, 2018), SHELXT2014/5 (Sheldrick, 2015a), SHELXL2018/3 (Sheldrick, 2015b) and ORTEP-3 for Windows (Farrugia, 2012).

\section{Acknowledgements}

Professor Nahossé Ziao is thanked for allowing the synthesis to be undertaken in the Laboratory of Thermodynamics and Physical Chemistry of the Environment (LTPCM), University Nangui, Abrogoua, Côte d'Ivoire.

\section{Funding information}

$\mathrm{TH}$ is grateful to Hacettepe University Scientific Research Project Unit (grant No. 013 D04 602 004).

\section{References}

Alam, M. \& Lee, D.-U. (2016). Comput. Biol. Chem. 64, 185-201. Bazinet, P., Yap, G. P. A. \& Richeson, D. S. (2003). J. Am. Chem. Soc. 125, 13314-13315.

Becke, A. D. (1993). J. Chem. Phys. 98, 5648-5652.

Behbahani, F. K. \& Golchin, F. M. (2017). J. Taibah Univ. Sci. 11, 8589.

Booysen, I. N., Ebinumoliseh, I., Sithebe, S., Akerman, M. P. \& Xulu, B. (2016). Polyhedron, 117, 755-760.

Bu, X., Deady, L. W., Finlay, G. J., Baguley, B. C. \& Denny, W. A. (2001). J. Med. Chem. 44, 2004-2014.

Cucciolito, M. E., Panunzi, B., Ruffo, F. \& Tuzi, A. (2013). Acta Cryst. E69, o1133-o1134.
De Aguiar, A. (1874). Ber. Dtsch. Chem. Ges. 7, 309-319.

Farrugia, L. J. (2012). J. Appl. Cryst. 45, 849-854.

Frisch, M. J., Trucks, G. W., Schlegel, H. B., Scuseria, G. E., Robb, M. A., Cheeseman, J. R., Scalmani, G., Barone, V., Mennucci, B., Petersson, G. A., Nakatsuji, H., Caricato, M., Li, X., Hratchian, H. P., Izmaylov, A. F., Bloino, J., Zheng, G., Sonnenberg, J. L., Hada, M., Ehara, M., Toyota, K., Fukuda, R., Hasegawa, J., Ishida, M., Nakajima, T., Honda, Y., Kitao, O., Nakai, H., Vreven, T., Montgomery, J. A. Jr, Peralta, J. E., Ogliaro, F., Bearpark, M., Heyd, J. J., Brothers, E., Kudin, K. N., Staroverov, V. N., Kobayashi, R., Normand, J., Raghavachari, K., Rendell, A., Burant, J. C., Iyengar, S. S., Tomasi, J., Cossi, M., Rega, N., Millam, J. M., Klene, M., Knox, J. E., Cross, J. B., Bakken, V., Adamo, C., Jaramillo, J., Gomperts, R., Stratmann, R. E., Yazyev, O., Austin, A. J., Cammi, R., Pomelli, C., Ochterski, J. W., Martin, R. L., Morokuma, K., Zakrzewski, V. G., Voth, G. A., Salvador, P., Dannenberg, J. J., Dapprich, S., Daniels, A. D., Farkas, Ö., Foresman, J. B., Ortiz, J. V., Cioslowski, J. \& Fox, D. J. (2009). GAUSSIAN09. Gaussian Inc., Wallingford, CT, US

Fun, H.-K., Chanawanno, K. \& Chantrapromma, S. (2011). Acta Cryst. E67, o715-o716.

Ghorbani, M. H. (2012). Acta Cryst. E68, o2605.

Giani, A. M., Lamperti, M., Maspero, A., Cimino, A., Negri, R., Giovenzana, G. B., Palmisano, G. \& Nardo, L. (2016). J. Lumin. 179, 384-392.

Hathwar, V. R., Sist, M., Jørgensen, M. R. V., Mamakhel, A. H., Wang, X., Hoffmann, C. M., Sugimoto, K., Overgaard, J. \& Iversen, B. B. (2015). IUCrJ, 2, 563-574.

He, X., Mao, J., Ma, Q. \& Tang, Y. (2018). J. Mol. Liq. 269, 260-268. Hirshfeld, H. L. (1977). Theor. Chim. Acta, 44, 129-138.

Mahapatra, A. K., Maji, R., Maiti, K., Manna, S. K., Mondal, S., Das Mukhopadhyay, C., Goswami, S., Sarkar, D., Mondal, T. K., Quah, C. K. \& Fun, H. K. (2015). Sens. Actuators B Chem. 207, 878-886.

Maloney, S., Slawin, A. M. Z. \& Woollins, J. D. (2013). Acta Cryst. E69, o246.

Manimekalai, A., Vijayalakshmi, N. \& Selvanayagam, S. (2014). Acta Cryst. E70, o959.

McKinnon, J. J., Jayatilaka, D. \& Spackman, M. A. (2007). Chem. Commun. pp. 3814-3816.

Rigaku OD (2018). CrysAlis PRO. Rigaku Oxford Diffraction, Yarnton, England.

Sheldrick, G. M. (2015a). Acta Cryst. A71, 3-8.

Sheldrick, G. M. (2015b). Acta Cryst. C71, 3-8.

Spackman, M. A. \& Jayatilaka, D. (2009). CrystEngComm, 11, 19-32.

Starshikoy, N. M. \& Pozharskii, F. T. (1973). Chem. Heterocycl. Compd. 9, 922-924.

Turner, M. J., McKinnon, J. J., Wolff, S. K., Grimwood, D. J., Spackman, P. R., Jayatilaka, D. \& Spackman, M. A. (2017). CrystalExplorer17. The University of Western Australia.

Varsha, G., Arun, V., Robinson, P. P., Sebastian, M., Varghese, D., Leeju, P., Jayachandran, V. P. \& Yusuff, K. M. M. (2010). Tetrahedron Lett. 51, 2174-2177.

Venkatesan, P., Thamotharan, S., Ilangovan, A., Liang, H. \& Sundius, T. (2016). Spectrochim. Acta A Mol. Biomol. Spectrosc. 153, 625636.

Watanab, K. \& Hareda, H. (1977). Chem. Abstr. 8499.

Woodgate, P. D., Herbert, J. M. \& Denny, W. A. (1987). Heterocycles, 26, 1029-1036.

Zhang, H. G., Wang, X. Z., Cao, Q., Gong, G. H. \& Quan, Z. S. (2017). Bioorg. Med. Chem. Lett. 27, 4409-4414.

Zhou, D. C., Lu, Y. T., Mai, Y. W., Zhang, C., Xia, J., Yao, P. F., Wang, H. G., Huang, S. L. \& Huang, Z. S. (2019). Bioorg. Chem. 91, 103131. 


\section{supporting information}

Acta Cryst. (2020). E76, 798-802 [https://doi.org/10.1107/S2056989020005939]

Crystal structure, Hirshfeld surface analysis and DFT studies of 2-(2,3-dihydro-1 $H$-perimidin-2-yl)phenol

Ballo Daouda, Nanou Tiéba Tuo, Niameke Jean-Baptiste Kangah, Tuncer Hökelek, Charles

Guillaume Kodjo, Pascal Retailleau and El Mokhtar Essassi

Computing details

Data collection: CrysAlis PRO (Rigaku OD, 2018); cell refinement: CrysAlis PRO (Rigaku OD, 2018); data reduction: CrysAlis PRO 1.171.39.46 (Rigaku OD, 2018); program(s) used to solve structure: SHELXT2014/5 (Sheldrick, 2015a); program(s) used to refine structure: SHELXL2018/3 (Sheldrick, 2015b); molecular graphics: ORTEP-3 for Windows (Farrugia, 2012).

2-(2,3-Dihydro-1 H-perimidin-2-yl)phenol

Crystal data

$\mathrm{C}_{17} \mathrm{H}_{14} \mathrm{~N}_{2} \mathrm{O}$

$M_{r}=262.30$

Monoclinic, $P 2_{1} / c$

$a=9.0710(4) \AA$

$b=12.0526(7) \AA$

$c=24.6120(11) \AA$

$\beta=95.999(4)^{\circ}$

$V=2676.1(2) \AA^{3}$

$Z=8$

$F(000)=1104$

$D_{\mathrm{x}}=1.302 \mathrm{Mg} \mathrm{m}^{-3}$

Mo $K \alpha$ radiation, $\lambda=0.71073 \AA$

Cell parameters from 8291 reflections

$\theta=2.8-29.0^{\circ}$

$\mu=0.08 \mathrm{~mm}^{-1}$

$T=293 \mathrm{~K}$

Plate, colourless

$0.60 \times 0.35 \times 0.05 \mathrm{~mm}$

\section{Data collection}

Rigaku XtaLAB PRO diffractometer

Radiation source: micro-focus sealed X-ray tube, Rigaku micromax 003

Rigaku Integrated Confocal MaxFlux double bounce multi-layer mirror optics monochromator

Detector resolution: 5.811 pixels $\mathrm{mm}^{-1}$ $\omega$ scans

$$
\begin{aligned}
& \text { Absorption correction: multi-scan } \\
& \quad \text { (CrysAlisPro; Rigaku OD, 2018) } \\
& T_{\min }=0.212, T_{\max }=1.000 \\
& 29344 \text { measured reflections } \\
& 6395 \text { independent reflections } \\
& 4554 \text { reflections with } I>2 \sigma(I) \\
& R_{\text {int }}=0.042 \\
& \theta_{\max }=29.4^{\circ}, \theta_{\min }=2.7^{\circ} \\
& h=-12 \rightarrow 11 \\
& k=-15 \rightarrow 16 \\
& l=-33 \rightarrow 32
\end{aligned}
$$

\section{Refinement}

Refinement on $F^{2}$

Least-squares matrix: full

6395 reflections

$R\left[F^{2}>2 \sigma\left(F^{2}\right)\right]=0.046$

379 parameters

$w R\left(F^{2}\right)=0.120$

0 restraints

$S=1.03$

Primary atom site location: other 
Secondary atom site location: difference Fourier map

Hydrogen site location: mixed

$\mathrm{H}$ atoms treated by a mixture of independent and constrained refinement

$$
\begin{aligned}
& w=1 /\left[\sigma^{2}\left(F_{\mathrm{o}}^{2}\right)+(0.0558 P)^{2}+0.390 P\right] \\
& \text { where } P=\left(F_{\mathrm{o}}^{2}+2 F_{\mathrm{c}}{ }^{2}\right) / 3 \\
& (\Delta / \sigma)_{\max }=0.001 \\
& \Delta \rho_{\max }=0.19 \mathrm{e} \AA^{-3} \\
& \Delta \rho_{\min }=-0.21 \mathrm{e} \AA^{-3}
\end{aligned}
$$

Special details

Geometry. All esds (except the esd in the dihedral angle between two 1.s. planes) are estimated using the full covariance

\begin{tabular}{|c|c|c|c|c|}
\hline & $x$ & $y$ & $z$ & $U_{\text {iso }} * / U_{\text {eq }}$ \\
\hline $\mathrm{C} 1 \mathrm{~A}$ & $0.56889(14)$ & $0.53907(11)$ & $0.62210(5)$ & $0.0396(3)$ \\
\hline $\mathrm{H} 1 \mathrm{~A}$ & 0.638920 & 0.526271 & 0.595013 & $0.047^{*}$ \\
\hline N1A & 0.41985 (13) & $0.50532(11)$ & $0.60023(5)$ & $0.0437(3)$ \\
\hline H1N1 & $0.4106(19)$ & $0.4334(16)$ & $0.5970(7)$ & $0.066^{*}$ \\
\hline O1A & $0.43505(13)$ & $0.56864(10)$ & 0.72308 & 0.0584 \\
\hline H1OA & $0.438(2)$ & $0.6117(18)$ & $0.6953(9)$ & $0.088 *$ \\
\hline $\mathrm{C} 2 \mathrm{~A}$ & $0.61653(14)$ & $0.47595(12)$ & $0.67387(5)$ & 0.0405 \\
\hline $\mathrm{N} 2 \mathrm{~A}$ & $0.56102(14)$ & $0.65756(10)$ & $0.63467(4)$ & 0.0435 \\
\hline H1NA & $0.646(2)$ & $0.6821(15)$ & $0.6495(7)$ & $0.065^{*}$ \\
\hline O1B & $0.04282(12)$ & $0.30406(13)$ & $0.63319(5)$ & 0.0708 (4) \\
\hline H1OB & $0.043(3)$ & $0.318(2)$ & $0.6666(10)$ & $0.106^{*}$ \\
\hline N1B & $0.20034(13)$ & $0.37845(10)$ & $0.73446(4)$ & $0.0411(3)$ \\
\hline H1NB & $0.2567(17)$ & 0.4294 (14) & $0.7230(6)$ & $0.049 *$ \\
\hline C1B & $0.25725(13)$ & $0.26647(11)$ & $0.72676(5)$ & 0.0375 \\
\hline H1B & 0.347428 & 0.254276 & 0.751615 & $0.045^{*}$ \\
\hline C3A & $0.54903(15)$ & $0.49467(12)$ & $0.72131(5)$ & $0.0443(3)$ \\
\hline $\mathrm{C} 2 \mathrm{~B}$ & $0.28946(14)$ & $0.24906(11)$ & $0.66876(5)$ & $0.0369(3)$ \\
\hline $\mathrm{N} 2 \mathrm{~B}$ & $0.14081(13)$ & $0.19020(11)$ & $0.74018(5)$ & 0.0419 \\
\hline $\mathrm{H} 2 \mathrm{NB}$ & $0.1541(17)$ & $0.1222(14)$ & $0.7310(6)$ & $0.050^{*}$ \\
\hline $\mathrm{C} 4 \mathrm{~A}$ & $0.59490(18)$ & $0.43710(15)$ & $0.76890(6)$ & $0.0582(4)$ \\
\hline $\mathrm{H} 4 \mathrm{~A}$ & 0.550760 & 0.450973 & 0.800663 & $0.070^{*}$ \\
\hline $\mathrm{C} 3 \mathrm{~B}$ & $0.17999(14)$ & $0.26533(12)$ & $0.62532(5)$ & 0.0433 \\
\hline C5A & $0.70642(19)$ & $0.35908(16)$ & $0.76890(7)$ & $0.0661(5)$ \\
\hline $\mathrm{H} 5 \mathrm{~A}$ & 0.736838 & 0.320194 & 0.800742 & $0.079 *$ \\
\hline C4B & $0.20960(17)$ & $0.24421(13)$ & $0.57226(6)$ & $0.0503(4)$ \\
\hline H4B & 0.135102 & 0.252304 & 0.543578 & $0.060 *$ \\
\hline C6A & $0.77248(17)$ & $0.33852(16)$ & $0.72245(8)$ & $0.0645(5)$ \\
\hline H6A & 0.846957 & 0.285454 & 0.722639 & $0.077 *$ \\
\hline C5B & 0.34887 (19) & $0.21132(14)$ & $0.56196(6)$ & $0.0568(4)$ \\
\hline H5B & 0.368794 & 0.198052 & 0.526223 & $0.068 *$ \\
\hline C7A & $0.72813(15)$ & $0.39693(14)$ & $0.67520(6)$ & 0.0519 (4) \\
\hline H7A & 0.773844 & 0.383022 & 0.643784 & $0.062 *$ \\
\hline C6B & $0.45901(18)$ & $0.19793(14)$ & $0.60424(7)$ & $0.0582(4)$ \\
\hline H6B & 0.553804 & 0.177175 & 0.597066 & $0.070 *$ \\
\hline
\end{tabular}
matrix. The cell esds are taken into account individually in the estimation of esds in distances, angles and torsion angles; correlations between esds in cell parameters are only used when they are defined by crystal symmetry. An approximate (isotropic) treatment of cell esds is used for estimating esds involving l.s. planes.

Fractional atomic coordinates and isotropic or equivalent isotropic displacement parameters $\left(\AA^{2}\right)$ 


$\begin{array}{lllll}\text { C9A } & 0.35196(14) & 0.56636(11) & 0.55642(5) & 0.0378(3) \\ \text { C7B } & 0.42863(15) & 0.21533(12) & 0.65727(6) & 0.0456(3) \\ \text { H7B } & 0.502848 & 0.204217 & 0.685787 & 0.055^{*} \\ \text { C10A } & 0.38759(13) & 0.68071(11) & 0.55413(5) & 0.0348(3) \\ \text { C9B } & 0.15927(15) & 0.40010(13) & 0.78695(5) & 0.0444(3) \\ \text { C11A } & 0.49532(14) & 0.72763(11) & 0.59321(5) & 0.0379(3) \\ \text { C10B } & 0.10259(14) & 0.30998(13) & 0.81524(5) & 0.0455(3) \\ \text { C12A } & 0.52565(18) & 0.83872(12) & 0.59224(6) & 0.0494(4) \\ \text { H12A } & 0.597660 & 0.868995 & 0.617626 & 0.059^{*} \\ \text { C11B } & 0.09143(14) & 0.20261(12) & 0.79161(5) & 0.0422(3) \\ \text { C13A } & 0.44833(19) & 0.90655(13) & 0.55310(6) & 0.0559(4) \\ \text { H13A } & 0.468574 & 0.982195 & 0.553059 & 0.067^{*} \\ \text { C12B } & 0.02694(16) & 0.11725(16) & 0.81762(6) & 0.0578(4) \\ \text { H12B } & 0.018478 & 0.047355 & 0.801591 & 0.069^{*} \\ \text { C14A } & 0.34396(17) & 0.86426(13) & 0.51502(6) & 0.0509(4) \\ \text { H14A } & 0.293631 & 0.911237 & 0.489454 & 0.061^{*} \\ \text { C13B } & -0.0259(2) & 0.1357(2) & 0.86819(8) & 0.0763(6) \\ \text { H13B } & -0.069782 & 0.077655 & 0.885520 & 0.092^{*} \\ \text { C15A } & 0.31142(14) & 0.75013(12) & 0.51393(5) & 0.0407(3) \\ \text { C14B } & -0.0142(2) & 0.2368(2) & 0.89238(8) & 0.0829(7) \\ \text { H14B } & -0.048187 & 0.246358 & 0.926437 & 0.099^{*} \\ \text { C16A } & 0.20329(16) & 0.70134(14) & 0.47590(5) & 0.0508(4) \\ \text { H16A } & 0.152944 & 0.744820 & 0.448781 & 0.061^{*} \\ \text { C15B } & 0.04892(18) & 0.32860(18) & 0.86688(6) & 0.0644(5) \\ \text { C17A } & 0.17231(16) & 0.59168(15) & 0.47860(6) & 0.0552(4) \\ \text { H17A } & 0.100450 & 0.561244 & 0.453204 & 0.066^{*} \\ \text { C16B } & 0.0568(2) & 0.4373(2) & 0.88808(8) & 0.0871(7) \\ \text { H16B } & 0.023178 & 0.451434 & 0.921832 & 0.105^{*} \\ \text { C18A } & 0.24558(16) & 0.52286(13) & 0.51861(6) & 0.0505(4) \\ \text { H18A } & 0.222179 & 0.447803 & 0.519553 & 0.061^{*} \\ \text { C18B } & 0.1656(2) & 0.50467(16) & 0.80913(7) & 0.0639(4) \\ \text { H18B } & 0.204313 & 0.563464 & 0.790689 & 0.077^{*} \\ \text { C17B } & 0.1125(3) & 0.5215(2) & 0.86015(8) & 0.0844(6) \\ \text { H17B } & 0.115746 & 0.592467 & 0.875106 & 0.101^{*} \\ & & & & \end{array}$

Atomic displacement parameters $\left(\AA^{2}\right)$

\begin{tabular}{lllllll}
\hline & $U^{11}$ & $U^{22}$ & $U^{33}$ & $U^{12}$ & $U^{13}$ & $U^{23}$ \\
\hline C1A & $0.0369(6)$ & $0.0440(8)$ & $0.0368(6)$ & $-0.0004(6)$ & $-0.0008(5)$ & $0.0035(5)$ \\
N1A & $0.0475(6)$ & $0.0373(6)$ & $0.0431(6)$ & $-0.0065(5)$ & $-0.0108(5)$ & $0.0045(5)$ \\
O1A & $0.0697(7)$ & $0.0588(7)$ & $0.0487(6)$ & $0.0109(6)$ & $0.0153(5)$ & $0.0095(5)$ \\
C2A & $0.0347(6)$ & $0.0438(8)$ & $0.0409(7)$ & $-0.0056(6)$ & $-0.0055(5)$ & $0.0066(6)$ \\
N2A & $0.0470(6)$ & $0.0416(7)$ & $0.0385(6)$ & $-0.0103(5)$ & $-0.0113(5)$ & $0.0042(5)$ \\
O1B & $0.0442(6)$ & $0.1251(12)$ & $0.0413(6)$ & $0.0251(6)$ & $-0.0033(5)$ & $-0.0002(6)$ \\
N1B & $0.0451(6)$ & $0.0392(7)$ & $0.0390(6)$ & $-0.0011(5)$ & $0.0046(5)$ & $-0.0005(5)$ \\
C1B & $0.0320(6)$ & $0.0436(8)$ & $0.0360(6)$ & $0.0035(5)$ & $-0.0009(5)$ & $0.0022(5)$ \\
C3A & $0.0443(7)$ & $0.0458(8)$ & $0.0414(7)$ & $-0.0062(6)$ & $-0.0017(6)$ & $0.0052(6)$ \\
C2B & $0.0372(6)$ & $0.0352(7)$ & $0.0383(6)$ & $-0.0002(5)$ & $0.0039(5)$ & $0.0013(5)$
\end{tabular}




\begin{tabular}{|c|c|c|c|c|c|c|}
\hline $\mathrm{N} 2 \mathrm{~B}$ & $0.0443(6)$ & $0.0397(7)$ & $0.0424(6)$ & $-0.0002(5)$ & $0.0079(5)$ & $0.0026(5)$ \\
\hline $\mathrm{C} 4 \mathrm{~A}$ & $0.0635(10)$ & $0.0667(11)$ & $0.0422(8)$ & $-0.0147(8)$ & $-0.0045(7)$ & $0.0124(7)$ \\
\hline C3B & $0.0396(7)$ & $0.0510(9)$ & $0.0392(7)$ & $0.0017(6)$ & $0.0038(5)$ & $0.0024(6)$ \\
\hline $\mathrm{C} 5 \mathrm{~A}$ & $0.0567(9)$ & $0.0738(12)$ & $0.0624(10)$ & $-0.0109(9)$ & $-0.0196(8)$ & $0.0315(9)$ \\
\hline C4B & $0.0583(9)$ & $0.0533(9)$ & $0.0386(7)$ & $-0.0017(7)$ & $0.0010(6)$ & $-0.0004(6)$ \\
\hline C6A & $0.0413(8)$ & $0.0676(11)$ & $0.0809(12)$ & $0.0057(8)$ & $-0.0115(8)$ & $0.0250(9)$ \\
\hline $\mathrm{C} 5 \mathrm{~B}$ & $0.0715(10)$ & $0.0573(10)$ & $0.0441(8)$ & $0.0027(8)$ & $0.0179(7)$ & $-0.0076(7)$ \\
\hline C7A & $0.0364(7)$ & $0.0584(10)$ & $0.0594(9)$ & $0.0023(7)$ & $-0.0020(6)$ & $0.0109(7)$ \\
\hline $\mathrm{C} 6 \mathrm{~B}$ & $0.0520(9)$ & $0.0632(11)$ & $0.0622(9)$ & $0.0089(8)$ & $0.0195(7)$ & $-0.0082(8)$ \\
\hline C9A & $0.0369(6)$ & $0.0431(8)$ & $0.0325(6)$ & $-0.0025(6)$ & $-0.0001(5)$ & $0.0012(5)$ \\
\hline C7B & $0.0396(7)$ & $0.0461(8)$ & $0.0511(8)$ & $0.0034(6)$ & $0.0045(6)$ & $-0.0031(6)$ \\
\hline $\mathrm{C} 10 \mathrm{~A}$ & $0.0343(6)$ & $0.0415(7)$ & $0.0288(6)$ & $0.0002(5)$ & $0.0048(5)$ & $0.0022(5)$ \\
\hline C9B & $0.0421(7)$ & $0.0532(9)$ & $0.0364(7)$ & $0.0082(6)$ & $-0.0033(5)$ & $-0.0045(6)$ \\
\hline $\mathrm{C} 11 \mathrm{~A}$ & $0.0416(7)$ & $0.0405(8)$ & $0.0316(6)$ & $-0.0032(6)$ & $0.0032(5)$ & $0.0015(5)$ \\
\hline C10B & $0.0359(7)$ & $0.0647(10)$ & $0.0349(6)$ & $0.0115(6)$ & -0.0008 & $0.0031(6)$ \\
\hline $\mathrm{C} 12 \mathrm{~A}$ & $0.0647(9)$ & $0.0424(8)$ & $0.0401(7)$ & $-0.0106(7)$ & $0.0007(6)$ & $-0.0014(6)$ \\
\hline $\mathrm{C} 11 \mathrm{~B}$ & $0.0313(6)$ & $0.0564(9)$ & $0.0381(7)$ & $0.0073(6)$ & $-0.0007(5)$ & $0.0114(6)$ \\
\hline C13A & $0.0798(11)$ & $0.0378(8)$ & $0.0506(8)$ & $-0.0022(8)$ & $0.0099(8)$ & $0.0058(6)$ \\
\hline $\mathrm{C} 12 \mathrm{~B}$ & $0.0459(8)$ & $0.0708(11)$ & $0.0566(9)$ & $0.0013(8)$ & $0.0049(7)$ & $0.0234(8)$ \\
\hline $\mathrm{C} 14 \mathrm{~A}$ & $0.0613(9)$ & $0.0492(9)$ & $0.0427(8)$ & $0.0103(7)$ & $0.0082(7)$ & $0.0139(6)$ \\
\hline C13B & $0.0614(11)$ & $0.1090(18)$ & $0.0607(11)$ & $0.0030(11)$ & $0.0162(8)$ & $0.0329(11)$ \\
\hline $\mathrm{C} 15 \mathrm{~A}$ & $0.0390(7)$ & $0.0512(9)$ & $0.0327(6)$ & $0.0044(6)$ & $0.0072(5)$ & $0.0074(6)$ \\
\hline C14B & $0.0694(12)$ & $0.137(2)$ & $0.0460(9)$ & $0.0154(13)$ & $0.0225(8)$ & $0.0196(12)$ \\
\hline $\mathrm{C} 16 \mathrm{~A}$ & $0.0443(7)$ & $0.0714(11)$ & $0.0352(7)$ & $0.0037(7)$ & $-0.0030(6)$ & $0.0124(7)$ \\
\hline C15B & $0.0549(9)$ & $0.0997(15)$ & $0.0388(8)$ & $0.0167(9)$ & $0.0057(7)$ & $-0.0008(8)$ \\
\hline C17A & $0.0482(8)$ & $0.0757(12)$ & $0.0383(7)$ & $-0.0105(8)$ & $-0.0119(6)$ & $0.0019(7)$ \\
\hline $\mathrm{C} 16 \mathrm{~B}$ & $0.0949(15)$ & $0.122(2)$ & $0.0457(9)$ & $0.0253(14)$ & $0.0137(9)$ & $-0.0216(11)$ \\
\hline $\mathrm{C} 18 \mathrm{~A}$ & $0.0539(8)$ & $0.0520(9)$ & $0.0429(7)$ & $-0.0127(7)$ & $-0.0078(6)$ & $-0.0002(6)$ \\
\hline C18B & $0.0766(11)$ & $0.0603(11)$ & $0.0528(9)$ & $0.0090(9)$ & $-0.0019(8)$ & $-0.0138(8)$ \\
\hline C17B & $0.1031(16)$ & $0.0873(16)$ & $0.0611(11)$ & $0.0239(13)$ & $0.0011(11)$ & $-0.0309(11)$ \\
\hline
\end{tabular}

Geometric parameters $\left(A,{ }^{\circ}\right)$

\begin{tabular}{llll}
\hline $\mathrm{C} 1 \mathrm{~A}-\mathrm{N} 1 \mathrm{~A}$ & $1.4597(17)$ & $\mathrm{C} 6 \mathrm{~B}-\mathrm{C} 7 \mathrm{~B}$ & $1.378(2)$ \\
$\mathrm{C} 1 \mathrm{~A}-\mathrm{N} 2 \mathrm{~A}$ & $1.4646(19)$ & $\mathrm{C} 6 \mathrm{~B}-\mathrm{H} 6 \mathrm{~B}$ & 0.9300 \\
$\mathrm{C} 1 \mathrm{~A}-\mathrm{C} 2 \mathrm{~A}$ & $1.5079(17)$ & $\mathrm{C} 9 \mathrm{~A}-\mathrm{C} 18 \mathrm{~A}$ & $1.3728(18)$ \\
$\mathrm{C} 1 \mathrm{~A}-\mathrm{H} 1 \mathrm{~A}$ & 0.9800 & $\mathrm{C} 9 \mathrm{~A}-\mathrm{C} 10 \mathrm{~A}$ & $1.4181(19)$ \\
$\mathrm{N} 1 \mathrm{~A}-\mathrm{C} 9 \mathrm{~A}$ & $1.3944(17)$ & $\mathrm{C} 7 \mathrm{~B}-\mathrm{H} 7 \mathrm{~B}$ & 0.9300 \\
$\mathrm{~N} 1 \mathrm{~A}-\mathrm{H} 1 \mathrm{~N} 1$ & $0.873(19)$ & $\mathrm{C} 10 \mathrm{~A}-\mathrm{C} 11 \mathrm{~A}$ & $1.4154(17)$ \\
$\mathrm{O} 1 \mathrm{~A}-\mathrm{C} 3 \mathrm{~A}$ & $1.3693(18)$ & $\mathrm{C} 10 \mathrm{~A}-\mathrm{C} 15 \mathrm{~A}$ & $1.4189(18)$ \\
$\mathrm{O} 1 \mathrm{~A}-\mathrm{H} 1 \mathrm{OA}$ & $0.86(2)$ & $\mathrm{C} 9 \mathrm{~B}-\mathrm{C} 18 \mathrm{~B}$ & $1.372(2)$ \\
$\mathrm{C} 2 \mathrm{~A}-\mathrm{C} 7 \mathrm{~A}$ & $1.388(2)$ & $\mathrm{C} 9 \mathrm{~B}-\mathrm{C} 10 \mathrm{~B}$ & $1.416(2)$ \\
$\mathrm{C} 2 \mathrm{~A}-\mathrm{C} 3 \mathrm{~A}$ & $1.3923(19)$ & $\mathrm{C} 11 \mathrm{~A}-\mathrm{C} 12 \mathrm{~A}$ & $1.368(2)$ \\
$\mathrm{N} 2 \mathrm{~A}-\mathrm{C} 11 \mathrm{~A}$ & $1.4081(17)$ & $\mathrm{C} 10 \mathrm{~B}-\mathrm{C} 11 \mathrm{~B}$ & $1.418(2)$ \\
$\mathrm{N} 2 \mathrm{~A}-\mathrm{H} 1 \mathrm{NA}$ & $0.870(19)$ & $\mathrm{C} 10 \mathrm{~B}-\mathrm{C} 15 \mathrm{~B}$ & $1.426(2)$ \\
$\mathrm{O} 1 \mathrm{~B}-\mathrm{C} 3 \mathrm{~B}$ & $1.3616(17)$ & $\mathrm{C} 12 \mathrm{~A}-\mathrm{C} 13 \mathrm{~A}$ & $1.395(2)$ \\
$\mathrm{O} 1 \mathrm{~B}-\mathrm{H} 1 \mathrm{OB}$ & $0.84(2)$ & $\mathrm{C} 12 \mathrm{~A}-\mathrm{H} 12 \mathrm{~A}$ & 0.9300 \\
$\mathrm{~N} 1 \mathrm{~B}-\mathrm{C} 9 \mathrm{~B}$ & $1.4059(17)$ & $\mathrm{C} 11 \mathrm{~B}-\mathrm{C} 12 \mathrm{~B}$ & $1.374(2)$
\end{tabular}




\begin{tabular}{|c|c|c|c|}
\hline $\mathrm{N} 1 \mathrm{~B}-\mathrm{C} 1 \mathrm{~B}$ & $1.4644(18)$ & $\mathrm{C} 13 \mathrm{~A}-\mathrm{C} 14 \mathrm{~A}$ & $1.360(2)$ \\
\hline $\mathrm{N} 1 \mathrm{~B}-\mathrm{H} 1 \mathrm{NB}$ & $0.865(17)$ & $\mathrm{C} 13 \mathrm{~A}-\mathrm{H} 13 \mathrm{~A}$ & 0.9300 \\
\hline $\mathrm{C} 1 \mathrm{~B}-\mathrm{N} 2 \mathrm{~B}$ & $1.4638(17)$ & $\mathrm{C} 12 \mathrm{~B}-\mathrm{C} 13 \mathrm{~B}$ & $1.397(3)$ \\
\hline $\mathrm{C} 1 \mathrm{~B}-\mathrm{C} 2 \mathrm{~B}$ & $1.5013(17)$ & $\mathrm{C} 12 \mathrm{~B}-\mathrm{H} 12 \mathrm{~B}$ & 0.9300 \\
\hline $\mathrm{C} 1 \mathrm{~B}-\mathrm{H} 1 \mathrm{~B}$ & 0.9800 & $\mathrm{C} 14 \mathrm{~A}-\mathrm{C} 15 \mathrm{~A}$ & $1.406(2)$ \\
\hline $\mathrm{C} 3 \mathrm{~A}-\mathrm{C} 4 \mathrm{~A}$ & $1.387(2)$ & $\mathrm{C} 14 \mathrm{~A}-\mathrm{H} 14 \mathrm{~A}$ & 0.9300 \\
\hline $\mathrm{C} 2 \mathrm{~B}-\mathrm{C} 7 \mathrm{~B}$ & $1.3833(18)$ & $\mathrm{C} 13 \mathrm{~B}-\mathrm{C} 14 \mathrm{~B}$ & $1.356(3)$ \\
\hline $\mathrm{C} 2 \mathrm{~B}-\mathrm{C} 3 \mathrm{~B}$ & $1.3949(18)$ & $\mathrm{C} 13 \mathrm{~B}-\mathrm{H} 13 \mathrm{~B}$ & 0.9300 \\
\hline $\mathrm{N} 2 \mathrm{~B}-\mathrm{C} 11 \mathrm{~B}$ & $1.3942(17)$ & $\mathrm{C} 15 \mathrm{~A}-\mathrm{C} 16 \mathrm{~A}$ & $1.412(2)$ \\
\hline $\mathrm{N} 2 \mathrm{~B}-\mathrm{H} 2 \mathrm{NB}$ & $0.862(17)$ & $\mathrm{C} 14 \mathrm{~B}-\mathrm{C} 15 \mathrm{~B}$ & $1.421(3)$ \\
\hline $\mathrm{C} 4 \mathrm{~A}-\mathrm{C} 5 \mathrm{~A}$ & $1.381(3)$ & $\mathrm{C} 14 \mathrm{~B}-\mathrm{H} 14 \mathrm{~B}$ & 0.9300 \\
\hline $\mathrm{C} 4 \mathrm{~A}-\mathrm{H} 4 \mathrm{~A}$ & 0.9300 & $\mathrm{C} 16 \mathrm{~A}-\mathrm{C} 17 \mathrm{~A}$ & $1.354(2)$ \\
\hline $\mathrm{C} 3 \mathrm{~B}-\mathrm{C} 4 \mathrm{~B}$ & $1.3840(19)$ & $\mathrm{C} 16 \mathrm{~A}-\mathrm{H} 16 \mathrm{~A}$ & 0.9300 \\
\hline $\mathrm{C} 5 \mathrm{~A}-\mathrm{C} 6 \mathrm{~A}$ & $1.368(3)$ & $\mathrm{C} 15 \mathrm{~B}-\mathrm{C} 16 \mathrm{~B}$ & $1.410(3)$ \\
\hline $\mathrm{C} 5 \mathrm{~A}-\mathrm{H} 5 \mathrm{~A}$ & 0.9300 & $\mathrm{C} 17 \mathrm{~A}-\mathrm{C} 18 \mathrm{~A}$ & $1.401(2)$ \\
\hline $\mathrm{C} 4 \mathrm{~B}-\mathrm{C} 5 \mathrm{~B}$ & $1.373(2)$ & $\mathrm{C} 17 \mathrm{~A}-\mathrm{H} 17 \mathrm{~A}$ & 0.9300 \\
\hline $\mathrm{C} 4 \mathrm{~B}-\mathrm{H} 4 \mathrm{~B}$ & 0.9300 & $\mathrm{C} 16 \mathrm{~B}-\mathrm{C} 17 \mathrm{~B}$ & $1.353(3)$ \\
\hline $\mathrm{C} 6 \mathrm{~A}-\mathrm{C} 7 \mathrm{~A}$ & $1.383(2)$ & $\mathrm{C} 16 \mathrm{~B}-\mathrm{H} 16 \mathrm{~B}$ & 0.9300 \\
\hline C6A-H6A & 0.9300 & $\mathrm{C} 18 \mathrm{~A}-\mathrm{H} 18 \mathrm{~A}$ & 0.9300 \\
\hline $\mathrm{C} 5 \mathrm{~B}-\mathrm{C} 6 \mathrm{~B}$ & $1.375(2)$ & $\mathrm{C} 18 \mathrm{~B}-\mathrm{C} 17 \mathrm{~B}$ & $1.406(3)$ \\
\hline $\mathrm{C} 5 \mathrm{~B}-\mathrm{H} 5 \mathrm{~B}$ & 0.9300 & $\mathrm{C} 18 \mathrm{~B}-\mathrm{H} 18 \mathrm{~B}$ & 0.9300 \\
\hline $\mathrm{C} 7 \mathrm{~A}-\mathrm{H} 7 \mathrm{~A}$ & 0.9300 & $\mathrm{C} 17 \mathrm{~B}-\mathrm{H} 17 \mathrm{~B}$ & 0.9300 \\
\hline $\mathrm{N} 1 \mathrm{~A}-\mathrm{C} 1 \mathrm{~A}-\mathrm{N} 2 \mathrm{~A}$ & $106.61(11)$ & $\mathrm{N} 1 \mathrm{~A}-\mathrm{C} 9 \mathrm{~A}-\mathrm{C} 10 \mathrm{~A}$ & $117.35(11)$ \\
\hline $\mathrm{N} 1 \mathrm{~A}-\mathrm{C} 1 \mathrm{~A}-\mathrm{C} 2 \mathrm{~A}$ & $110.09(11)$ & $\mathrm{C} 6 \mathrm{~B}-\mathrm{C} 7 \mathrm{~B}-\mathrm{C} 2 \mathrm{~B}$ & $121.03(13)$ \\
\hline $\mathrm{N} 2 \mathrm{~A}-\mathrm{C} 1 \mathrm{~A}-\mathrm{C} 2 \mathrm{~A}$ & $109.23(11)$ & $\mathrm{C} 6 \mathrm{~B}-\mathrm{C} 7 \mathrm{~B}-\mathrm{H} 7 \mathrm{~B}$ & 119.5 \\
\hline $\mathrm{N} 1 \mathrm{~A}-\mathrm{C} 1 \mathrm{~A}-\mathrm{H} 1 \mathrm{~A}$ & 110.3 & $\mathrm{C} 2 \mathrm{~B}-\mathrm{C} 7 \mathrm{~B}-\mathrm{H} 7 \mathrm{~B}$ & 119.5 \\
\hline $\mathrm{N} 2 \mathrm{~A}-\mathrm{C} 1 \mathrm{~A}-\mathrm{H} 1 \mathrm{~A}$ & 110.3 & $\mathrm{C} 11 \mathrm{~A}-\mathrm{C} 10 \mathrm{~A}-\mathrm{C} 9 \mathrm{~A}$ & $120.35(11)$ \\
\hline $\mathrm{C} 2 \mathrm{~A}-\mathrm{C} 1 \mathrm{~A}-\mathrm{H} 1 \mathrm{~A}$ & 110.3 & $\mathrm{C} 11 \mathrm{~A}-\mathrm{C} 10 \mathrm{~A}-\mathrm{C} 15 \mathrm{~A}$ & $119.31(12)$ \\
\hline $\mathrm{C} 9 \mathrm{~A}-\mathrm{N} 1 \mathrm{~A}-\mathrm{C} 1 \mathrm{~A}$ & $117.08(11)$ & $\mathrm{C} 9 \mathrm{~A}-\mathrm{C} 10 \mathrm{~A}-\mathrm{C} 15 \mathrm{~A}$ & 120.30 \\
\hline $\mathrm{C} 9 \mathrm{~A}-\mathrm{N} 1 \mathrm{~A}-\mathrm{H} 1 \mathrm{~N} 1$ & $115.0(12)$ & $\mathrm{C} 18 \mathrm{~B}-\mathrm{C} 9 \mathrm{~B}-\mathrm{N} 1 \mathrm{~B}$ & $122.14(15)$ \\
\hline $\mathrm{C} 1 \mathrm{~A}-\mathrm{N} 1 \mathrm{~A}-\mathrm{H} 1 \mathrm{~N} 1$ & $112.7(12)$ & $\mathrm{C} 18 \mathrm{~B}-\mathrm{C} 9 \mathrm{~B}-\mathrm{C} 10 \mathrm{~B}$ & $120.75(14)$ \\
\hline $\mathrm{C} 3 \mathrm{~A}-\mathrm{O} 1 \mathrm{~A}-\mathrm{H} 1 \mathrm{OA}$ & $106.1(14)$ & $\mathrm{N} 1 \mathrm{~B}-\mathrm{C} 9 \mathrm{~B}-\mathrm{C} 10 \mathrm{~B}$ & $117.02(13)$ \\
\hline $\mathrm{C} 7 \mathrm{~A}-\mathrm{C} 2 \mathrm{~A}-\mathrm{C} 3 \mathrm{~A}$ & $118.36(13)$ & $\mathrm{C} 12 \mathrm{~A}-\mathrm{C} 11 \mathrm{~A}-\mathrm{N} 2 \mathrm{~A}$ & $121.94(12)$ \\
\hline $\mathrm{C} 7 \mathrm{~A}-\mathrm{C} 2 \mathrm{~A}-\mathrm{C} 1 \mathrm{~A}$ & $120.67(13)$ & $\mathrm{C} 12 \mathrm{~A}-\mathrm{C} 11 \mathrm{~A}-\mathrm{C} 10 \mathrm{~A}$ & $120.34(12)$ \\
\hline $\mathrm{C} 3 \mathrm{~A}-\mathrm{C} 2 \mathrm{~A}-\mathrm{C} 1 \mathrm{~A}$ & $120.97(12)$ & $\mathrm{N} 2 \mathrm{~A}-\mathrm{C} 11 \mathrm{~A}-\mathrm{C} 10 \mathrm{~A}$ & $117.56(12)$ \\
\hline $\mathrm{C} 11 \mathrm{~A}-\mathrm{N} 2 \mathrm{~A}-\mathrm{C} 1 \mathrm{~A}$ & $117.26(10)$ & $\mathrm{C} 9 \mathrm{~B}-\mathrm{C} 10 \mathrm{~B}-\mathrm{C} 11 \mathrm{~B}$ & $120.84(12)$ \\
\hline $\mathrm{C} 11 \mathrm{~A}-\mathrm{N} 2 \mathrm{~A}-\mathrm{H} 1 \mathrm{NA}$ & $112.9(12)$ & $\mathrm{C} 9 \mathrm{~B}-\mathrm{C} 10 \mathrm{~B}-\mathrm{C} 15 \mathrm{~B}$ & $119.55(15)$ \\
\hline $\mathrm{C} 1 \mathrm{~A}-\mathrm{N} 2 \mathrm{~A}-\mathrm{H} 1 \mathrm{NA}$ & $111.1(12)$ & $\mathrm{C} 11 \mathrm{~B}-\mathrm{C} 10 \mathrm{~B}-\mathrm{C} 15 \mathrm{~B}$ & $119.53(15)$ \\
\hline $\mathrm{C} 3 \mathrm{~B}-\mathrm{O} 1 \mathrm{~B}-\mathrm{H} 1 \mathrm{OB}$ & $107.7(17)$ & $\mathrm{C} 11 \mathrm{~A}-\mathrm{C} 12 \mathrm{~A}-\mathrm{C} 13 \mathrm{~A}$ & $119.88(14)$ \\
\hline $\mathrm{C} 9 \mathrm{~B}-\mathrm{N} 1 \mathrm{~B}-\mathrm{C} 1 \mathrm{~B}$ & $114.90(11)$ & $\mathrm{C} 11 \mathrm{~A}-\mathrm{C} 12 \mathrm{~A}-\mathrm{H} 12 \mathrm{~A}$ & 120.1 \\
\hline $\mathrm{C} 9 \mathrm{~B}-\mathrm{N} 1 \mathrm{~B}-\mathrm{H} 1 \mathrm{NB}$ & $113.0(10)$ & $\mathrm{C} 13 \mathrm{~A}-\mathrm{C} 12 \mathrm{~A}-\mathrm{H} 12 \mathrm{~A}$ & 120.1 \\
\hline $\mathrm{C} 1 \mathrm{~B}-\mathrm{N} 1 \mathrm{~B}-\mathrm{H} 1 \mathrm{NB}$ & $112.5(10)$ & $\mathrm{C} 12 \mathrm{~B}-\mathrm{C} 11 \mathrm{~B}-\mathrm{N} 2 \mathrm{~B}$ & $122.37(15)$ \\
\hline $\mathrm{N} 2 \mathrm{~B}-\mathrm{C} 1 \mathrm{~B}-\mathrm{N} 1 \mathrm{~B}$ & $106.09(10)$ & $\mathrm{C} 12 \mathrm{~B}-\mathrm{C} 11 \mathrm{~B}-\mathrm{C} 10 \mathrm{~B}$ & $120.51(14)$ \\
\hline $\mathrm{N} 2 \mathrm{~B}-\mathrm{C} 1 \mathrm{~B}-\mathrm{C} 2 \mathrm{~B}$ & $110.09(11)$ & $\mathrm{N} 2 \mathrm{~B}-\mathrm{C} 11 \mathrm{~B}-\mathrm{C} 10 \mathrm{~B}$ & $117.02(12)$ \\
\hline $\mathrm{N} 1 \mathrm{~B}-\mathrm{C} 1 \mathrm{~B}-\mathrm{C} 2 \mathrm{~B}$ & $110.99(10)$ & $\mathrm{C} 14 \mathrm{~A}-\mathrm{C} 13 \mathrm{~A}-\mathrm{C} 12 \mathrm{~A}$ & $121.31(14)$ \\
\hline $\mathrm{N} 2 \mathrm{~B}-\mathrm{C} 1 \mathrm{~B}-\mathrm{H} 1 \mathrm{~B}$ & 109.9 & $\mathrm{C} 14 \mathrm{~A}-\mathrm{C} 13 \mathrm{~A}-\mathrm{H} 13 \mathrm{~A}$ & 119.3 \\
\hline $\mathrm{N} 1 \mathrm{~B}-\mathrm{C} 1 \mathrm{~B}-\mathrm{H} 1 \mathrm{~B}$ & 109.9 & $\mathrm{C} 12 \mathrm{~A}-\mathrm{C} 13 \mathrm{~A}-\mathrm{H} 13 \mathrm{~A}$ & 119.3 \\
\hline
\end{tabular}




$\begin{array}{ll}\text { C2B-C1B-H1B } & 109.9 \\ \text { O1A-C3A-C4A } & 117.37(13) \\ \text { O1A-C3A-C2A } & 122.12(12) \\ \text { C4A-C3A-C2A } & 120.50(14) \\ \text { C7B-C2B-C3B } & 118.45(12) \\ \text { C7B-C2B-C1B } & 120.52(11) \\ \text { C3B-C2B-C1B } & 121.02(11) \\ \text { C11B-N2B-C1B } & 116.45(11) \\ \text { C11B-N2B-H2NB } & 114.1(10) \\ \text { C1B-N2B-H2NB } & 114.4(10) \\ \text { C5A-C4A-C3A } & 119.71(16) \\ \text { C5A-C4A-H4A } & 120.1 \\ \text { C3A-C4A-H4A } & 120.1 \\ \text { O1B-C3B-C4B } & 117.87(12) \\ \text { O1B-C3B-C2B } & 121.81(12) \\ \text { C4B-C3B-C2B } & 120.30(12) \\ \text { C6A-C5A-C4A } & 120.55(14) \\ \text { C6A-C5A-H5A } & 119.7 \\ \text { C4A-C5A-H5A } & 119.7 \\ \text { C5B-C4B-C3B } & 120.04(14) \\ \text { C5B-C4B-H4B } & 120.0 \\ \text { C3B-C4B-H4B } & 120.0 \\ \text { C5A-C6A-C7A } & 119.76(16) \\ \text { C5A-C6A-H6A } & 120.1 \\ \text { C7A-C6A-H6A } & 120.1 \\ \text { C4B-C5B-C6B } & 120.27(13) \\ \text { C4B-C5B-H5B } & 119.9 \\ \text { C6B-C5B-H5B } & 119.9 \\ \text { C6A-C7A-C2A } & 121.10(15) \\ \text { C6A-C7A-H7A } & 119.4 \\ \text { C2A-C7A-H7A } & 119.4 \\ \text { C5B-C6B-C7B } & 119.85(14) \\ \text { C5B-C6B-H6B } & 120.1 \\ \text { C7B-C6B-H6B } & 120.1 \\ \text { C18A-C9A-N1A } & 123.03(13) \\ \text { C18A-C9A-C10A } & 119.42(12) \\ \text { N2A-C1A-N1A-C9A } & 53.40(15) \\ \text { C2A-C1A-N1A-C9A } & 171.78(12) \\ \text { N1A-C1A-C2A-C7A } & 110.84(15) \\ \text { N2A-C1A-C2A-C7A } & -132.41(14) \\ \text { N1A-C1A-C2A-C3A } & -68.59(16) \\ \text { N2A-C1A-C2A-C3A } & 48.16(16) \\ \text { N1A-C1A-N2A-C11A } & -50.96(15) \\ \text { C2A-C1A-N2A-C11A } & -169.89(11) \\ \text { C9B-N1B-C1B-N2B } & 57.04(13) \\ \text { C9B-N1B-C1B-C2B } & 176.61) \\ \text { C7A-C2A-C3A-O1A } & -177.96(13) \\ & \end{array}$

$\begin{array}{ll}\text { C11B-C12B-C13B } & 119.89(19) \\ \text { C11B-C12B-H12B } & 120.1 \\ \text { C13B-C12B-H12B } & 120.1 \\ \text { C13A-C14A-C15A } & 120.62(13) \\ \text { C13A-C14A-H14A } & 119.7 \\ \text { C15A-C14A-H14A } & 119.7 \\ \text { C14B-C13B-C12B } & 121.03(18) \\ \text { C14B-C13B-H13B } & 119.5 \\ \text { C12B-C13B-H13B } & 119.5 \\ \text { C14A-C15A-C16A } & 123.37(13) \\ \text { C14A-C15A-C10A } & 118.52(12) \\ \text { C16A-C15A-C10A } & 118.08(13) \\ \text { C13B-C14B-C15B } & 121.50(16) \\ \text { C13B-C14B-H14B } & 119.3 \\ \text { C15B-C14B-H14B } & 119.3 \\ \text { C17A-C16A-C15A } & 120.50(13) \\ \text { C17A-C16A-H16A } & 119.8 \\ \text { C15A-C16A-H16A } & 119.8 \\ \text { C16B-C15B-C14B } & 124.57(18) \\ \text { C16B-C15B-C10B } & 117.87(18) \\ \text { C14B-C15B-C10B } & 117.52(18) \\ \text { C16A-C17A-C18A } & 121.78(13) \\ \text { C16A-C17A-H17A } & 119.1 \\ \text { C18A-C17A-H17A } & 119.1 \\ \text { C17B-C16B-C15B } & 121.10(17) \\ \text { C17B-C16B-H16B } & 119.4 \\ \text { C15B-C16B-H16B } & 119.4 \\ \text { C9A-C18A-C17A } & 119.91(14) \\ \text { C9A-C18A-H18A } & 120.0 \\ \text { C17A-C18A-H18A } & 120.0 \\ \text { C9B-C18B-C17B } & 118.95(19) \\ \text { C9B-C18B-H18B } & 120.5 \\ \text { C17B-C18B-H18B } & 120.5 \\ \text { C16B-C17B-C18B } & 121.76(19) \\ \text { C16B-C17B-H17B } & 119.1 \\ \text { C18B-C17B-H17B } & 119.1 \\ \text { C1A-N2A-C11A-C10A } & 27.40(17) \\ \text { C9A-C10A-C11A-C12A } & -177.51(12) \\ \text { C15A-C10A-C11A-C12A } & 0.02(18) \\ \text { C9A-C10A-C11A-N2A } & -1.84(18) \\ \text { C15A-C10A-C11A-N2A } & 175.69(11) \\ \text { C18B-C9B-C10B-C11B } & 177.89(14) \\ \text { N1B-C9B-C10B-C11B } & 1.32(18) \\ \text { C18B-C9B-C10B-C15B } & 1.2(2) \\ \text { N1B-C9B-C10B-C15B } & -175.39(12) \\ \text { N2A-C11A-C12A-C13A } & -174.43(13) \\ \text { C10A-C11A-C12A-C13A } & 1.0(2) \\ & \end{array}$




$\begin{array}{ll}\mathrm{C} 1 \mathrm{~A}-\mathrm{C} 2 \mathrm{~A}-\mathrm{C} 3 \mathrm{~A}-\mathrm{O} 1 \mathrm{~A} & 1.5(2) \\ \mathrm{C} 7 \mathrm{~A}-\mathrm{C} 2 \mathrm{~A}-\mathrm{C} 3 \mathrm{~A}-\mathrm{C} 4 \mathrm{~A} & 1.3(2) \\ \mathrm{C} 1 \mathrm{~A}-\mathrm{C} 2 \mathrm{~A}-\mathrm{C} 3 \mathrm{~A}-\mathrm{C} 4 \mathrm{~A} & -179.23(13) \\ \mathrm{N} 2 \mathrm{~B}-\mathrm{C} 1 \mathrm{~B}-\mathrm{C} 2 \mathrm{~B}-\mathrm{C} 7 \mathrm{~B} & -118.22(14) \\ \mathrm{N} 1 \mathrm{~B}-\mathrm{C} 1 \mathrm{~B}-\mathrm{C} 2 \mathrm{~B}-\mathrm{C} 7 \mathrm{~B} & 124.63(13) \\ \mathrm{N} 2 \mathrm{~B}-\mathrm{C} 1 \mathrm{~B}-\mathrm{C} 2 \mathrm{~B}-\mathrm{C} 3 \mathrm{~B} & 61.14(16) \\ \mathrm{N} 1 \mathrm{~B}-\mathrm{C} 1 \mathrm{~B}-\mathrm{C} 2 \mathrm{~B}-\mathrm{C} 3 \mathrm{~B} & -56.01(16) \\ \mathrm{N} 1 \mathrm{~B}-\mathrm{C} 1 \mathrm{~B}-\mathrm{N} 2 \mathrm{~B}-\mathrm{C} 11 \mathrm{~B} & -55.21(14) \\ \mathrm{C} 2 \mathrm{~B}-\mathrm{C} 1 \mathrm{~B}-\mathrm{N} 2 \mathrm{~B}-\mathrm{C} 11 \mathrm{~B} & -175.37(11) \\ \mathrm{O} 1 \mathrm{~A}-\mathrm{C} 3 \mathrm{~A}-\mathrm{C} 4 \mathrm{~A}-\mathrm{C} 5 \mathrm{~A} & 178.05(14) \\ \mathrm{C} 2 \mathrm{~A}-\mathrm{C} 3 \mathrm{~A}-\mathrm{C} 4 \mathrm{~A}-\mathrm{C} 5 \mathrm{~A} & -1.3(2) \\ \mathrm{C} 7 \mathrm{~B}-\mathrm{C} 2 \mathrm{~B}-\mathrm{C} 3 \mathrm{~B}-\mathrm{O} 1 \mathrm{~B} & -176.49(14) \\ \mathrm{C} 1 \mathrm{~B}-\mathrm{C} 2 \mathrm{~B}-\mathrm{C} 3 \mathrm{~B}-\mathrm{O} 1 \mathrm{~B} & 4.1(2) \\ \mathrm{C} 7 \mathrm{~B}-\mathrm{C} 2 \mathrm{~B}-\mathrm{C} 3 \mathrm{~B}-\mathrm{C} 4 \mathrm{~B} & 2.2(2) \\ \mathrm{C} 1 \mathrm{~B}-\mathrm{C} 2 \mathrm{~B}-\mathrm{C} 3 \mathrm{~B}-\mathrm{C} 4 \mathrm{~B} & -177.14(13) \\ \mathrm{C} 3 \mathrm{~A}-\mathrm{C} 4 \mathrm{~A}-\mathrm{C} 5 \mathrm{~A}-\mathrm{C} 6 \mathrm{~A} & 0.3(3) \\ \mathrm{O} 1 \mathrm{~B}-\mathrm{C} 3 \mathrm{~B}-\mathrm{C} 4 \mathrm{~B}-\mathrm{C} 5 \mathrm{~B} & 176.17(15) \\ \mathrm{C} 2 \mathrm{~B}-\mathrm{C} 3 \mathrm{~B}-\mathrm{C} 4 \mathrm{~B}-\mathrm{C} 5 \mathrm{~B} & -2.6(2) \\ \mathrm{C} 4 \mathrm{~A}-\mathrm{C} 5 \mathrm{~A}-\mathrm{C} 6 \mathrm{~A}-\mathrm{C} 7 \mathrm{~A} & 0.5(3) \\ \mathrm{C} 3 \mathrm{~B}-\mathrm{C} 4 \mathrm{~B}-\mathrm{C} 5 \mathrm{~B}-\mathrm{C} 6 \mathrm{~B} & 0.8(2) \\ \mathrm{C} 5 \mathrm{~A}-\mathrm{C} 6 \mathrm{~A}-\mathrm{C} 7 \mathrm{~A}-\mathrm{C} 2 \mathrm{~A} & -0.4(3) \\ \mathrm{C} 3 \mathrm{~A}-\mathrm{C} 2 \mathrm{~A}-\mathrm{C} 7 \mathrm{~A}-\mathrm{C} 6 \mathrm{~A} & -0.5(2) \\ \mathrm{C} 1 \mathrm{~A}-\mathrm{C} 2 \mathrm{~A}-\mathrm{C} 7 \mathrm{~A}-\mathrm{C} 6 \mathrm{~A} & -179.92(14) \\ \mathrm{C} 4 \mathrm{~B}-\mathrm{C} 5 \mathrm{~B}-\mathrm{C} 6 \mathrm{~B}-\mathrm{C} 7 \mathrm{~B} & 1.4(3) \\ \mathrm{C} 1 \mathrm{~A}-\mathrm{N} 1 \mathrm{~A}-\mathrm{C} 9 \mathrm{~A}-\mathrm{C} 18 \mathrm{~A} & 153.25(13) \\ \mathrm{C} 1 \mathrm{~A}-\mathrm{N} 1 \mathrm{~A}-\mathrm{C} 9 \mathrm{~A}-\mathrm{C} 10 \mathrm{~A} & -31.88(17) \\ \mathrm{C} 5 \mathrm{~B}-\mathrm{C} 6 \mathrm{~B}-\mathrm{C} 7 \mathrm{~B}-\mathrm{C} 2 \mathrm{~B} & -1.7(2) \\ \mathrm{C} 3 \mathrm{~B}-\mathrm{C} 2 \mathrm{~B}-\mathrm{C} 7 \mathrm{~B}-\mathrm{C} 6 \mathrm{~B} & -0.1(2) \\ \mathrm{C} 1 \mathrm{~B}-\mathrm{C} 2 \mathrm{~B}-\mathrm{C} 7 \mathrm{~B}-\mathrm{C} 6 \mathrm{~B} & 179.29(14) \\ \mathrm{C} 18 \mathrm{~A}-\mathrm{C} 9 \mathrm{~A}-\mathrm{C} 10 \mathrm{~A}-\mathrm{C} 11 \mathrm{~A} & 179.05(12) \\ \mathrm{N} 1 \mathrm{~A}-\mathrm{C} 9 \mathrm{~A}-\mathrm{C} 10 \mathrm{~A}-\mathrm{C} 11 \mathrm{~A} & 3.99(17) \\ \mathrm{C} 18 \mathrm{~A}-\mathrm{C} 9 \mathrm{~A}-\mathrm{C} 10 \mathrm{~A}-\mathrm{C} 15 \mathrm{~A} & 1.53(19) \\ \mathrm{N} 1 \mathrm{~A}-\mathrm{C} 9 \mathrm{~A}-\mathrm{C} 10 \mathrm{~A}-\mathrm{C} 15 \mathrm{~A} & -173.53(11) \\ \mathrm{C} 1 \mathrm{~B}-\mathrm{N} 1 \mathrm{~B}-\mathrm{C} 9 \mathrm{~B}-\mathrm{C} 18 \mathrm{~B} & 151.26(14) \\ \mathrm{C} 1 \mathrm{~B}-\mathrm{N} 1 \mathrm{~B}-\mathrm{C} 9 \mathrm{~B}-\mathrm{C} 10 \mathrm{~B} & -32.21(16) \\ \mathrm{C} 1 \mathrm{~A}-\mathrm{N} 2 \mathrm{~A}-\mathrm{C} 11 \mathrm{~A}-\mathrm{C} 12 \mathrm{~A} & -157.00(13) \\ & \\ & \end{array}$

\begin{tabular}{|c|c|}
\hline $\mathrm{C} 1 \mathrm{~B}-\mathrm{N} 2 \mathrm{~B}-\mathrm{C} 11 \mathrm{~B}-\mathrm{C} 12 \mathrm{~B}$ & $-155.68(12)$ \\
\hline $\mathrm{C} 1 \mathrm{~B}-\mathrm{N} 2 \mathrm{~B}-\mathrm{C} 11 \mathrm{~B}-\mathrm{C} 10 \mathrm{~B}$ & $27.86(16)$ \\
\hline $\mathrm{C} 9 \mathrm{~B}-\mathrm{C} 10 \mathrm{~B}-\mathrm{C} 11 \mathrm{~B}-\mathrm{C} 12 \mathrm{~B}$ & $-175.50(12)$ \\
\hline $\mathrm{C} 15 \mathrm{~B}-\mathrm{C} 10 \mathrm{~B}-\mathrm{C} 11 \mathrm{~B}-\mathrm{C} 12 \mathrm{~B}$ & $1.21(19)$ \\
\hline $\mathrm{C} 9 \mathrm{~B}-\mathrm{C} 10 \mathrm{~B}-\mathrm{C} 11 \mathrm{~B}-\mathrm{N} 2 \mathrm{~B}$ & $1.03(18)$ \\
\hline $\mathrm{C} 15 \mathrm{~B}-\mathrm{C} 10 \mathrm{~B}-\mathrm{C} 11 \mathrm{~B}-\mathrm{N} 2 \mathrm{~B}$ & $177.74(12)$ \\
\hline $\mathrm{C} 11 \mathrm{~A}-\mathrm{C} 12 \mathrm{~A}-\mathrm{C} 13 \mathrm{~A}-\mathrm{C} 14 \mathrm{~A}$ & $-0.9(2)$ \\
\hline $\mathrm{N} 2 \mathrm{~B}-\mathrm{C} 11 \mathrm{~B}-\mathrm{C} 12 \mathrm{~B}-\mathrm{C} 13 \mathrm{~B}$ & $-177.43(13)$ \\
\hline $\mathrm{C} 10 \mathrm{~B}-\mathrm{C} 11 \mathrm{~B}-\mathrm{C} 12 \mathrm{~B}-\mathrm{C} 13 \mathrm{~B}$ & $-1.1(2)$ \\
\hline $\mathrm{C} 12 \mathrm{~A}-\mathrm{C} 13 \mathrm{~A}-\mathrm{C} 14 \mathrm{~A}-\mathrm{C} 15 \mathrm{~A}$ & $-0.3(2)$ \\
\hline $\mathrm{C} 11 \mathrm{~B}-\mathrm{C} 12 \mathrm{~B}-\mathrm{C} 13 \mathrm{~B}-\mathrm{C} 14 \mathrm{~B}$ & $-0.3(3)$ \\
\hline $\mathrm{C} 13 \mathrm{~A}-\mathrm{C} 14 \mathrm{~A}-\mathrm{C} 15 \mathrm{~A}-\mathrm{C} 16 \mathrm{~A}$ & $179.26(14)$ \\
\hline $\mathrm{C} 13 \mathrm{~A}-\mathrm{C} 14 \mathrm{~A}-\mathrm{C} 15 \mathrm{~A}-\mathrm{C} 10 \mathrm{~A}$ & $1.3(2)$ \\
\hline $\mathrm{C} 11 \mathrm{~A}-\mathrm{C} 10 \mathrm{~A}-\mathrm{C} 15 \mathrm{~A}-\mathrm{C} 14 \mathrm{~A}$ & $-1.20(18)$ \\
\hline $\mathrm{C} 9 \mathrm{~A}-\mathrm{C} 10 \mathrm{~A}-\mathrm{C} 15 \mathrm{~A}-\mathrm{C} 14 \mathrm{~A}$ & $176.34(12)$ \\
\hline $\mathrm{C} 11 \mathrm{~A}-\mathrm{C} 10 \mathrm{~A}-\mathrm{C} 15 \mathrm{~A}-\mathrm{C} 16 \mathrm{~A}$ & $-179.24(11)$ \\
\hline $\mathrm{C} 9 \mathrm{~A}-\mathrm{C} 10 \mathrm{~A}-\mathrm{C} 15 \mathrm{~A}-\mathrm{C} 16 \mathrm{~A}$ & $-1.70(18)$ \\
\hline $\mathrm{C} 12 \mathrm{~B}-\mathrm{C} 13 \mathrm{~B}-\mathrm{C} 14 \mathrm{~B}-\mathrm{C} 15 \mathrm{~B}$ & $1.5(3)$ \\
\hline $\mathrm{C} 14 \mathrm{~A}-\mathrm{C} 15 \mathrm{~A}-\mathrm{C} 16 \mathrm{~A}-\mathrm{C} 17 \mathrm{~A}$ & $-176.90(14)$ \\
\hline $\mathrm{C} 10 \mathrm{~A}-\mathrm{C} 15 \mathrm{~A}-\mathrm{C} 16 \mathrm{~A}-\mathrm{C} 17 \mathrm{~A}$ & $1.0(2)$ \\
\hline $\mathrm{C} 13 \mathrm{~B}-\mathrm{C} 14 \mathrm{~B}-\mathrm{C} 15 \mathrm{~B}-\mathrm{C} 16 \mathrm{~B}$ & $176.13(19)$ \\
\hline $\mathrm{C} 13 \mathrm{~B}-\mathrm{C} 14 \mathrm{~B}-\mathrm{C} 15 \mathrm{~B}-\mathrm{C} 10 \mathrm{~B}$ & $-1.3(3)$ \\
\hline $\mathrm{C} 9 \mathrm{~B}-\mathrm{C} 10 \mathrm{~B}-\mathrm{C} 15 \mathrm{~B}-\mathrm{C} 16 \mathrm{~B}$ & $-0.9(2)$ \\
\hline $\mathrm{C} 11 \mathrm{~B}-\mathrm{C} 10 \mathrm{~B}-\mathrm{C} 15 \mathrm{~B}-\mathrm{C} 16 \mathrm{~B}$ & $-177.64(15)$ \\
\hline $\mathrm{C} 9 \mathrm{~B}-\mathrm{C} 10 \mathrm{~B}-\mathrm{C} 15 \mathrm{~B}-\mathrm{C} 14 \mathrm{~B}$ & $176.72(14)$ \\
\hline $\mathrm{C} 11 \mathrm{~B}-\mathrm{C} 10 \mathrm{~B}-\mathrm{C} 15 \mathrm{~B}-\mathrm{C} 14 \mathrm{~B}$ & $0.0(2)$ \\
\hline $\mathrm{C} 15 \mathrm{~A}-\mathrm{C} 16 \mathrm{~A}-\mathrm{C} 17 \mathrm{~A}-\mathrm{C} 18 \mathrm{~A}$ & $-0.2(2)$ \\
\hline $\mathrm{C} 14 \mathrm{~B}-\mathrm{C} 15 \mathrm{~B}-\mathrm{C} 16 \mathrm{~B}-\mathrm{C} 17 \mathrm{~B}$ & $-176.93(19)$ \\
\hline $\mathrm{C} 10 \mathrm{~B}-\mathrm{C} 15 \mathrm{~B}-\mathrm{C} 16 \mathrm{~B}-\mathrm{C} 17 \mathrm{~B}$ & $0.5(3)$ \\
\hline $\mathrm{N} 1 \mathrm{~A}-\mathrm{C} 9 \mathrm{~A}-\mathrm{C} 18 \mathrm{~A}-\mathrm{C} 17 \mathrm{~A}$ & $174.10(13)$ \\
\hline $\mathrm{C} 10 \mathrm{~A}-\mathrm{C} 9 \mathrm{~A}-\mathrm{C} 18 \mathrm{~A}-\mathrm{C} 17 \mathrm{~A}$ & $-0.7(2)$ \\
\hline $\mathrm{C} 16 \mathrm{~A}-\mathrm{C} 17 \mathrm{~A}-\mathrm{C} 18 \mathrm{~A}-\mathrm{C} 9 \mathrm{~A}$ & $0.0(2)$ \\
\hline $\mathrm{N} 1 \mathrm{~B}-\mathrm{C} 9 \mathrm{~B}-\mathrm{C} 18 \mathrm{~B}-\mathrm{C} 17 \mathrm{~B}$ & $175.37(15)$ \\
\hline $\mathrm{C} 10 \mathrm{~B}-\mathrm{C} 9 \mathrm{~B}-\mathrm{C} 18 \mathrm{~B}-\mathrm{C} 17 \mathrm{~B}$ & $-1.0(2)$ \\
\hline $\mathrm{C} 15 \mathrm{~B}-\mathrm{C} 16 \mathrm{~B}-\mathrm{C} 17 \mathrm{~B}-\mathrm{C} 18 \mathrm{~B}$ & $-0.4(3)$ \\
\hline $\mathrm{C} 9 \mathrm{~B}-\mathrm{C} 18 \mathrm{~B}-\mathrm{C} 17 \mathrm{~B}-\mathrm{C} 16 \mathrm{~B}$ & $0.6(3)$ \\
\hline
\end{tabular}

Hydrogen-bond geometry $\left(\AA,{ }^{\circ}\right)$

\begin{tabular}{lllll}
\hline$D-\mathrm{H} \cdots A$ & $D-\mathrm{H}$ & $\mathrm{H} \cdots A$ & $D \cdots A$ & $D-\mathrm{H} \cdots A$ \\
\hline $\mathrm{O} 1 A-\mathrm{H} 1 O A \cdots \mathrm{N} 1 A$ & $0.86(2)$ & $2.66(2)$ & $3.1072(16)$ & $113.8(17)$ \\
$\mathrm{O} 1 A-\mathrm{H} 1 O A \cdots \mathrm{N} 2 A$ & $0.86(2)$ & $2.03(2)$ & $2.7763(16)$ & $144.6(19)$ \\
$\mathrm{O} 1 B-\mathrm{H} 1 O B \cdots \mathrm{N} 1 B$ & $0.84(2)$ & $2.20(3)$ & $2.8835(16)$ & $138(2)$ \\
$\mathrm{O} 1 B-\mathrm{H} 1 O B \cdots \mathrm{N} 2 B$ & $0.84(2)$ & $2.47(2)$ & $3.0196(16)$ & $123(2)$ \\
$\mathrm{N} 1 B-\mathrm{H} 1 N B \cdots \mathrm{O} 1 A$ & $0.865(17)$ & $2.331(17)$ & $3.1608(18)$ & $160.8(14)$ \\
\hline
\end{tabular}

